\title{
Interval-valued Pythagorean Fuzzy Frank Power Aggregation Operators based on An Isomorphic Frank Dual Triple
}

\author{
Yi Yang ${ }^{2,3}$, Zhen-Song Chen ${ }^{1,3}$, Yue-Hua Chen ${ }^{1}$, Kwai-Sang Chin ${ }^{3}$ \\ ${ }^{1}$ School of Civil Engineering, Wuhan University, Wuhan 430072, China \\ E-mail: zschen@whu.edu.cn \& yuhchen@whu.edu.cn \\ ${ }^{2}$ Institute of Big Data and Internet Innovation, Key Laboratory of Hunan Province for New Retail Virtual \\ Reality Technology, Hunan University of Commerce, Changsha 410205, China \\ ${ }^{3}$ Department of Systems Engineering and Engineering Management, City University of Hong Kong,Kowloon \\ Tong, Hong Kong, China \\ E-mail:yangshijiazu@my.swjtu.edu.cn \& mekschin@cityu.edu.hk
}

Received 12 March 2018

Accepted 23 May 2018

\begin{abstract}
Interval-valued Pythagorean fuzzy sets (PFSs), as an extension of PFSs, have strong potential in the management of complex uncertainty in real-world applications. This study aims to develop several intervalvalued Pythagorean fuzzy Frank power (IVPFFP) aggregation operators with an adjustable parameter via the integration of an isomorphic Frank dual triple. First, a special automorphism on unit interval is introduced to construct an isomorphic Frank dual triple; and this triple is further applied on the definition of interval-valued Pythagorean fuzzy Frank operational laws. Second, two IVPFFP aggregation operators with the inclusion of an adjustable parameter are defined on the basis of the proposed operational laws, and several instrumental properties are then investigated. Furthermore, some limiting cases of the proposed IVPFFP operators are analyzed with respect to the varying adjustable parameter values. Finally, an IVPFFP aggregation operator-based multiple attribute group decision-making model is developed with a practical example furnished to demonstrate its feasibility and efficiency. The power that the adjustable parameter exhibits has been leveraged to affect the final decision results, and the proposed IVPFFP operators are compared with three selected aggregation operators to demonstrate their advantages provided with a practical example.
\end{abstract}

Keywords: Interval-valued Pythagorean fuzzy numbers; Frank dual triple; Frank power operators.

\section{Introduction}

The term Pythagorean fuzzy set $(\mathrm{PFS})^{1,2}$ was coined by Yager as a powerful extension of intuitionistic fuzzy set (IFS) ${ }^{3}$. PFS, akin to IFS, is composed of membership grade $\mu$ and nonmembership grade $v$ and is further delivered to form a binary group repre- sentation. The core distinction between IFS and PFS is reflected in the constraint of the grade pairs, which is $\mu+v \leqslant 1$ for IFS and is $\mu^{2}+v^{2} \leqslant 1$ for PFS. PFSs include IFSs as a whole and pose few barriers of information representation. A plethora of practical applications of PFS have demonstrated its utility in addressing multiple attribute group decision-making

\footnotetext{
* Corresponding author.
} 
(MAGDM) problems ${ }^{4-9,36}$. The main characteristic of the membership and nonmembership degrees of PFS is that their values are often expressed as real numbers. However, in certain cases, decision makers (DMs) may only be able to provide a range of values for these grades. Consequently, PFS is not applicable to these cases. In view of this deficiency, the notion of interval-valued PFS (IVPFS) was further developed ${ }^{10,11}$. IVPFS enables DMs to express their uncertainty via the provision of interval-valued membership and nonmembership values. Several researchers have conducted related studies on the application of IVPFS in MAGDM ${ }^{10-13}$.

In developing various fuzzy sets such as IFS, hesitant fuzzy set (HFS) and hesitant-intuitionistic (or dual hesitant) fuzzy set, the basic operations for them play an indispensable role, which is also not an exception for IVPFS. However, few studies on the operations for IVPFS have been conducted ${ }^{10,11}$, especially the generalized operations. In fact, some generalized operations on various types of extended fuzzy sets have been developed on the basis of the Frank dual triple, such as intuitionistic Frank operations ${ }^{14,15}$, interval-valued intuitionistic Frank operations ${ }^{16}$, hesitant Frank operations ${ }^{17}$, triangular interval type-2 fuzzy Frank operations ${ }^{18}$, interval intuitionistic linguistic Frank operations ${ }^{19}$ and dual hesitant fuzzy Frank operations ${ }^{20}$. The Frank dual triple consists of a standard negation, Frank t-norm, and its dual s-norm ${ }^{21}$ with the adjustable parameter $\chi$. A desirable feature of this triple is that DMs can select different values to obtain various types of dual triple. In the cases of $\chi \rightarrow 1$ and $\chi \rightarrow \infty$, for example, then Frank dual triple will reduce to the algebraic dual triple and the Lukasiewicz dual triple, respectively. However, a numerical example will be provided to reveal that the Frank dual triple is not suitable for defining the generalized operations on IVPFSs. In view of the reasons mentioned before, an automorphism on $[0,1]$ will be introduced in this study to develop an isomorphic Frank dual triple, which includes an isomorphic Frank t-norm, an isomorphic Frank s-norm and the Pythagorean negation $^{1,2}$. Then, this new dual triple can be used to define the Frank operations on IVPFSs.

A core step of MAGDM is to aggregate multi- ple assessment matrixes into a synthesis assessment matrix, which is often performed by appropriately selecting aggregation operators ${ }^{31,32}$. Recently, some aggregation operators have been proposed to fuse multiple interval-valued Pythagorean fuzzy numbers (IVPFNs), such as IVPFWA and IVPFWG aggregation operators $^{10,12,13}$. To deal with the correlation among the input arguments in MAGDM problems, many studies ${ }^{22-25}$ have used power average (PA) to successfully model such situation ${ }^{26}$. Thus, in this study, the PA and power geometric (PG) operators ${ }^{27}$ were extended to IVPFSs. Inspired by their research, the Frank operational laws will be used to propose the interval-valued Pythagorean fuzzy Frank power weighted average (IVPFFPWA) and interval-valued Pythagorean fuzzy Frank power weighted geometric (IVPFFPWG) aggregation operators. A prominent feature of the aggregation weights for the two aggregation operators is that they not only consider the importance of experts but also depend upon the supports from the remaining input IVPFNs. Moreover, the relationships between the Pythagorean Frank aggregation operators and their related adjustable parameters will be analyzed, and some limiting cases of these operators will as well be investigated. Finally, by applying the proposed Frank aggregation operators, a novel decision making approach is constructed to deal with the MAGDM problem with IVPFNs. With the numerical example provided, the relationship between the proposed aggregation operators and their adjustable parameters can be explained accordingly.

The rest of paper is structured as follows. Relevant definitions of IVPFSs are reviewed in Section 2. Section 3 proposes an isomorphic Frank dual triple, which is then used to define the Frank operations for IVPFSs. Subsequently, the IVPFFPWA and IVPFFPWG aggregation operators are developed in Section 4. Section 5 applies the proposed aggregation operators to develop a simple decision-making approach to solving MAGDM with IVPFNs. An illustrative example is provided to verify the proposed approach in Section 6. Finally, section 7 concludes this paper. 


\section{Preliminaries}

Relevant definitions of IVPFSs are reviewed and the dual triple, which consists of t-norm, s-norm, and negation, is introduced along with its components. Particular attention will be paid to the Frank dual triple. We then provide the definition of isomorphism dual triple, which is essential in this study.

\subsection{Related definitions of IVPFSs}

Definition 1. ${ }^{3}$ Let $K$ denote a finite universal set, and then a IFS $B$ on $K$ is provided as

$$
B=\left\{\left\langle q, \mu_{B}(q), v_{B}(q)\right\rangle \mid q \in K\right\},
$$

where $\mu_{B}: K \rightarrow[0,1]$ is the membership function , $v_{B}: K \rightarrow[0,1]$ is the nonmembership function of $B$, and $\mu_{B}(q)+\mu_{B}(q) \leqslant 1$. We call the two tuples $\left(\mu_{B}(q), \mu_{B}(q)\right)$ as intuitionistic fuzzy number (IFN) and simply express it as $B=\left(\mu_{B}, v_{B}\right)$, where $\mu_{B}, v_{B} \in[0,1]$ and $\mu_{B}+v_{B} \leqslant 1$.

Definition 2. ${ }^{1,2}$ Given a finite universal set $K$, a PFS $P$ on $K$ is defined as

$$
P=\left\{\left\langle y, \mu_{P}(y), v_{P}(y)\right\rangle \mid y \in K\right\},
$$

where $\mu_{P}: K \rightarrow[0,1]$ is the membership function, $v_{P}: K \rightarrow[0,1]$ is the nonmembership function of $P$ and $\mu_{P}^{2}(y)+\mu_{P}^{2}(y) \leqslant 1$. We call the two tuples $\left(\mu_{P}(y), v_{P}(y)\right)$ as Pythagorean fuzzy number
(PFN) and simply express it as $\beta=\left(\mu_{P}, v_{P}\right)$, where $\mu_{P}, v_{P} \in[0,1]$ and $\mu_{P}^{2}+\mu_{P}^{2} \leqslant 1$.

Definition 3. ${ }^{10,11}$ Given a finite universal set $K$, and IVPFS $\widetilde{P}$ on $K$ is provided by

$$
\widetilde{P}=\left\{\left\langle p, \widetilde{\mu}_{\widetilde{P}}(p), \widetilde{v}_{\widetilde{P}}(p)\right\rangle \mid p \in K\right\},
$$

where $\widetilde{\mu}_{\widetilde{P}}(p): K \rightarrow \varepsilon([0,1])$ and $\widetilde{v}_{\widetilde{P}}(p): K \rightarrow$ $\varepsilon([0,1])$ are the membership and nonmembership functions, respectively. In addition, $\sup \left(\widetilde{\mu}_{\widetilde{P}}^{2}(p)\right)+$ $\sup \left(\widetilde{v}_{\widetilde{P}}^{2}(p)\right) \leqslant 1 . \quad \varepsilon([0,1])$ is the set of all closed intervals in $[0,1]$, and we call the two tuples $\left(\widetilde{\mu}_{\widetilde{P}}(p), \widetilde{v}_{\widetilde{P}}(p)\right)$ as interval-valued Pythagorean fuzzy number (IVPFN). If we let $\widetilde{\mu}_{\widetilde{P}}(p)=\left[p^{-}, p^{+}\right]$ and $\widetilde{v}_{\widetilde{P}}(p)=\left[q^{-}, q^{+}\right]$, then IVPFN can be expressed as $\widetilde{P}=\left(\left[p^{-}, p^{+}\right],\left[q^{-}, q^{+}\right]\right)$, where $\left(p^{+}\right)^{2}+\left(q^{+}\right)^{2} \leqslant$ 1.

Definition 4. ${ }^{10,11}$ Let $\widetilde{P}_{l}=\left(\left[p_{l}^{-}, p_{l}^{+}\right],\left[q_{l}^{-}, q_{l}^{+}\right]\right)(l=1,2)$ be two IVPFNs, and their natural partial order relation are provided as follows:

(1) $\widetilde{P}_{1}=\widetilde{P}_{2}$ iff $p_{1}^{-}=p_{2}^{-}, p_{1}^{+}=p_{2}^{+}, q_{1}^{-}=q_{2}^{-}$and $q_{1}^{+}=q_{2}^{+}$.

(2) $\widetilde{P}_{1} \leqslant \widetilde{P}_{2}$ iff $p_{1}^{-} \leqslant p_{2}^{-}, p_{1}^{+} \leqslant p_{2}^{+}, q_{1}^{-} \geqslant q_{2}^{-}$and $q_{1}^{+} \geqslant q_{2}^{+}$.

Definition 5. ${ }^{10,11}$. Let $\widetilde{P}_{l}=\left(\left[p_{l}^{-}, p_{l}^{+}\right],\left[q_{l}^{-}, q_{l}^{+}\right]\right)(l=1,2)$ be two IVPFNs, some fundamental operations are provided:

(1) $\widetilde{P}_{l}^{c}=\left(\left[q_{l}^{-}, q_{l}^{+}\right],\left[p_{l}^{-}, p_{l}^{+}\right]\right)$.

(2) $\widetilde{P}_{1} \oplus \widetilde{P}_{2}=\left(\left[\sqrt{\left(p_{1}^{-}\right)^{2}+\left(p_{2}^{-}\right)^{2}-\left(p_{1}^{-} p_{2}^{-}\right)^{2}}, \sqrt{\left(p_{1}^{+}\right)^{2}+\left(p_{2}^{+}\right)^{2}-\left(p_{1}^{+} p_{2}^{+}\right)^{2}}\right],\left[q_{1}^{-} q_{2}^{-}, q_{1}^{+} q_{2}^{+}\right]\right)$;

(3) $\widetilde{P}_{1} \otimes \widetilde{P}_{2}=\left(\left[p_{1}^{-} p_{2}^{-}, p_{1}^{+} p_{2}^{+}\right],\left[\sqrt{\left(q_{1}^{-}\right)^{2}+\left(q_{2}^{-}\right)^{2}-\left(q_{1}^{-} q_{2}^{-}\right)^{2}}, \sqrt{\left(q_{1}^{+}\right)^{2}+\left(q_{2}^{+}\right)^{2}-\left(q_{1}^{+} q_{2}^{+}\right)^{2}}\right]\right)$;

(4) $\kappa \widetilde{P}_{1}=\left(\left[\sqrt{1-\left(1-\left(p_{1}^{-}\right)^{2}\right)^{\kappa}}, \sqrt{1-\left(1-\left(p_{1}^{+}\right)^{2}\right)^{\kappa}}\right],\left[\left(q_{1}^{-}\right)^{\kappa},\left(q_{1}^{+}\right)^{\kappa}\right]\right), \kappa>0$;

(5) $\widetilde{P}_{1}^{\kappa}=\left(\left[\left(p_{1}^{-}\right)^{\kappa},\left(p_{1}^{+}\right)^{\kappa}\right],\left[\sqrt{1-\left(1-\left(q_{1}^{-}\right)^{2}\right)^{\kappa}}, \sqrt{1-\left(1-\left(q_{1}^{+}\right)^{2}\right)^{\kappa}}\right]\right), \kappa>0$.

Definition 6. ${ }^{10,11}$ Let $\widetilde{P}_{l}=\left(\left[p_{l}^{-}, p_{l}^{+}\right],\left[q_{l}^{-}, q_{l}^{+}\right]\right)(l=1,2)$ vided as follows: be two IVPFNs, then their distance measure is pro-

$$
\begin{aligned}
d\left(\widetilde{P}_{1}, \widetilde{P}_{2}\right) & =\frac{1}{4}\left(\left|\left(p_{1}^{-}\right)^{2}-\left(p_{2}^{-}\right)^{2}\right|+\left|\left(p_{1}^{+}\right)^{2}-\left(p_{2}^{+}\right)^{2}\right|\right) \\
& +\frac{1}{4}\left(\left|\left(q_{1}^{-}\right)^{2}-\left(q_{2}^{-}\right)^{2}\right|+\left|\left(q_{1}^{+}\right)^{2}-\left(q_{2}^{+}\right)^{2}\right|\right) \\
& +\frac{1}{4}\left(\left|\left(\pi_{1}^{-}\right)^{2}-\left(\pi_{2}^{-}\right)^{2}\right|+\left|\left(\pi_{1}^{+}\right)^{2}-\left(\pi_{2}^{+}\right)^{2}\right|\right)
\end{aligned}
$$


where

$\tilde{\pi}\left(\widetilde{P}_{l}\right)=\left[\sqrt{1-\left(p_{l}^{+}\right)^{2}-\left(q_{l}^{+}\right)^{2}}, \sqrt{1-\left(p_{l}^{-}\right)^{2}-\left(q_{l}^{-}\right)^{2}}\right]$

Definition 7. 10 Given two IVPFNs $\widetilde{P}_{l}=$ $\left(\left[p_{l}^{-}, p_{l}^{+}\right],\left[q_{l}^{-}, q_{l}^{+}\right]\right), l=1,2$, a ranking method of them is provided as follows:

(1) if $\operatorname{Sco}\left(\widetilde{P}_{1}\right)<\operatorname{Sco}\left(\widetilde{P}_{2}\right)$, then $\widetilde{\alpha}_{1} \prec \widetilde{\alpha}_{2}$.

(2) if $\operatorname{Sco}\left(\widetilde{P}_{1}\right)=\operatorname{Sco}\left(\widetilde{P}_{2}\right) \wedge \operatorname{Acc}\left(\widetilde{P}_{1}\right)<\operatorname{Acc}\left(\widetilde{P}_{2}\right)$, then $\widetilde{P}_{1} \prec \widetilde{P}_{2}$.

(3) if $\operatorname{Sco}\left(\widetilde{P}_{1}\right)=\operatorname{Sco}\left(\widetilde{P}_{2}\right) \wedge \operatorname{Acc}\left(\widetilde{P}_{1}\right)=\operatorname{Acc}\left(\widetilde{P}_{2}\right)$, then $\widetilde{P}_{1}^{\sim} \widetilde{P}_{2}$.

where

$$
\operatorname{Sco}\left(\widetilde{P}_{l}\right)=\frac{1}{2}\left(\left(p_{l}^{-}\right)^{2}+\left(p_{l}^{+}\right)^{2}-\left(q_{l}^{-}\right)^{2}-\left(q_{l}^{+}\right)^{2}\right)
$$

is the score function of $\widetilde{P}_{l}(l=1,2)$, and

$$
\operatorname{Acc}\left(\widetilde{P}_{l}\right)=\frac{1}{2}\left(\left(p_{l}^{-}\right)^{2}+\left(p_{l}^{+}\right)^{2}+\left(q_{l}^{-}\right)^{2}+\left(q_{l}^{+}\right)^{2}\right)
$$

is the accuracy function of $\widetilde{P}_{l}(l=1,2)$.

\subsection{Frank dual triple}

Definition 8. ${ }^{28}$ A continuous function $\wp:[c, d] \rightarrow$ $[c, d]$ is called an automorphism on $[c, d]$ iff the following conditions are satisfied:

(i) $\wp$ is a strictly monotonic increasing function.

(ii) $\wp(c)=c$ and $\wp(d)=d$.

Definition 9. ${ }^{28,29}$ A negation $\eta$ is a mapping on the $[0,1]$, which satisfies the following conditions:

(1) $\eta$ is a monotonic decreasing function;

(2) $\eta(0)=1$ and $\eta(1)=0$.

In particular, a continuous negation that is a strictly decreasing function is called a strict negation, and a strict negation that satisfies $\eta(\eta(x))=x$ is called a strong negation.

Remark 1. The classical negation $\eta(x)=1-x$, also known as Zadeh negation, is an important and frequently used negation. The Pythagorean negation $\eta(x)=\sqrt{1-x^{2}}$ is another negation, which is required and has been used in $\mathrm{PFSs}^{1,2}$. It is worth mentioning that the Zadeh and Pythagorean negations both belong to the renowned Yager negation. In the rest of this study, the classical negation and Pythagorean negation are simply denoted as $\eta_{I}$ and $\eta_{P}$, respectively.

Remark 2. To facilitate our further discussion, the following general symbols are employed throughout this study:

(1) $N=\{1,2, \cdots, n\}$ and $N_{4}=\{1,2,3,4\}$.

(2) $M=\{1,2, \cdots, m\}$ and $M_{4}=\{1,2,3,4\}$.

(3) $T=\{1,2, \cdots, t\}$ and $T_{3}=\{1,2,3\}$.

(4) $\widetilde{\mathbb{P}}=\left(\widetilde{P}_{1}, \cdots, \widetilde{P}_{n}\right)$, where

$$
\widetilde{P}_{l}=\left(\left[p_{l}^{-}, p_{l}^{+}\right],\left[q_{l}^{-}, q_{l}^{+}\right]\right)(l \in N)
$$

are $n$ IVPFNs.

(5) $\widetilde{\mathbb{Q}}=\left(\widetilde{Q}_{1}, \widetilde{Q}_{2}, \cdots, \widetilde{Q}_{n}\right)$, where

$$
\widetilde{Q}_{l}=\left(\left[m_{l}^{-}, m_{l}^{+}\right],\left[n_{l}^{-}, n_{l}^{+}\right]\right)(l \in N)
$$

are $n$ IVPFNs.

(6) $W=\left(\omega_{1}, \omega_{2}, \cdots, \omega_{n}\right)$ is the weighting vector, where $\sum_{l=1}^{n} \omega_{l}=1$ and $\omega_{l} \geqslant 0(l \in N)$.

(7) $U=\left(u_{1}, u_{2}, \cdots, u_{n}\right)$ is the weighting vector, where $\sum_{l=1}^{n} u_{l}=1$ and $u_{l} \geqslant 0(l \in N)$.

(8) $\wp$ is an automorphism on $[0,1]$, and $\wp(x)=x^{2}$.

Theorem 1. ${ }^{28,29}$ Let $\eta$ be a negation. Then, $\eta$ is a strong negation iff there exists an automorphism $\phi$ from $[0,1]$ to $[0,1]$ such that

$$
\eta=\phi^{-1} \circ \eta_{I} \circ \phi .
$$

Definition 10. ${ }^{28}$ A t-norm $X$ is a binary operation on the unit interval that satisfies at least the following axioms for any $p_{1}, p_{2}, p_{3} \in[0,1]$ :

(i) $X\left(p_{1}, 1\right)=1$.

(ii) if $p_{1} \leqslant p_{2}$ then $X\left(p_{1}, p_{3}\right) \leqslant X\left(p_{2}, p_{3}\right)$.

(iii) $X\left(p_{1}, p_{2}\right)=X\left(p_{2}, p_{1}\right)$.

(iv) $X\left(p_{1},\left(p_{2}, p_{3}\right)\right)=X\left(\left(p_{1}, p_{2}\right), p_{3}\right)$.

An Archimedean t-norm $T$ satisfies the following conditions:

(v) $X$ is an continuous function;

(vi) $X\left(p_{1}, p_{1}\right)<p_{1}$.

Definition 11. ${ }^{28}$ A s-norm $Y$ is a mapping $Y$ : $[0,1]^{2} \rightarrow[0,1]$ that satisfies the following conditions for any $p_{1}, p_{2}, p_{3} \in[0,1]$ : 
(i) $Y\left(p_{1}, 0\right)=0$;

(ii) if $p_{1} \leqslant p_{2}$ then $Y\left(p_{1}, p_{3}\right) \leqslant Y\left(p_{2}, p_{3}\right)$;

(iii) $Y\left(p_{1}, p_{2}\right)=Y\left(p_{2}, p_{1}\right)$;

(iv) $Y\left(p_{1},\left(p_{2}, p_{3}\right)\right)=Y\left(\left(p_{1}, p_{2}\right), p_{3}\right)$.

An Archimedean s-norm $Y$ satisfies the following conditions:

(v) $Y$ is an continuous function;

(vi) $Y\left(p_{1}, p_{1}\right)>p_{1}$.

Definition 12. ${ }^{28} \mathrm{~A}$ t-norm $X$ and a s-norm $Y$ are dual with respect to the negation $\eta$, if the following conditions are satisfied:

(i) For any $p, q \in[0,1], Y(p, q)=\eta(X(\eta(p), \eta(q)))$.

(ii) For any $p, q \in[0,1], \quad X(p, q)=$ $\eta(Y(\eta(p), \eta(q)))$.

Moreover, the triple $(X, Y, \eta)$ is called a dual triple.

Theorem 2. ${ }^{28}$ Given a strong negation $\eta$, then (1) Let $X$ be an Archimedean t-norm. If $Y$ satisfies the condition ( $i$ ) in Definition 12, then $(X, Y, \eta)$ is a dual triple;

(2) Let $Y$ be an Archimedean s-norm. If $X$ satisfies the condition (ii) in Definition 12, then $(X, Y, \eta)$ is a dual triple.

Definition 13. ${ }^{21}$ The Frank t-norm $X_{F}$ is provided as

$$
\begin{aligned}
X_{F}(p, q) & =\hbar_{F}^{(-1)}\left(\hbar_{F}(p)+\hbar_{F}(q)\right) \\
& =\frac{\ln \left(1+\left(\chi^{p}-1\right)\left(\chi^{q}-1\right) /(\chi-1)\right)}{\ln \chi}
\end{aligned}
$$

Then, $\hbar_{F}$ is the decreasing generator such that $\hbar_{F}(t)=\ln \left((\chi-1) /\left(\chi^{t}-1\right)\right)$ and $\chi \in(1, \infty) . \hbar_{F}^{(-1)}$ is the pseudo-inverse of $\hbar_{F}$.

Definition 14. ${ }^{21}$ The Frank s-norm $Y_{F}$ is provided as

$$
\begin{aligned}
Y_{F}(p, q) & =g_{F}^{(-1)}\left(g_{F}(p)+g_{F}(q)\right) \\
& =1-\frac{\ln \left(1+\left(\chi^{1-p}-1\right)\left(\chi^{1-q}-1\right) /(\chi-1)\right)}{\ln \chi}
\end{aligned}
$$

where $g_{F}$ is the increasing generator which is given by $g_{F}=\hbar_{F} \circ \eta_{I}, g_{F}^{(-1)}$ is the pseudo-inverse of $g_{F}$.

Theorem 3. Let $X_{F}$ and $Y_{F}$ be the Frank t-norm and s-norm, respectively. Then, the dual $\left(X_{F}, Y_{F}, \eta_{I}\right)$ is a dual triple which is called Frank dual triple.
Some limiting cases of the Frank dual triple are provided as following.

Case 1. If $\chi \rightarrow 1$, then the Frank dual triple reduces to Algebraic dual triple $\left(X_{A}, Y_{A}, \eta_{I}\right)$, where

$$
X_{A}(p, q)=p q
$$

is the Algebraic t-norm, and

$$
Y_{A}(p, a)=1-(1-p)(1-q)
$$

is the Algebraic s-norm.

Case 2. If $\chi \rightarrow \infty$, then the Frank dual triple reduces to Lukasiewicz dual triple $\left(X_{L}, Y_{L}, \eta_{I}\right)$, where

$$
Y_{L}(p, q)=\min \{0, p+q-1\}
$$

is called the Lukasiewicz s-norm, and

$$
X_{L}(p, q)=\min \{p+q, 1\}
$$

is called the Lukasiewicz t-norm.

Definition 15. ${ }^{29}$ Given a t-norm $X$ and an automorphism $\phi$ on $[0,1]$, the function

$$
X_{\phi}(p, q)=\phi^{-1}(X(\phi(p), \phi(q)))
$$

is also a t-norm and is called as an isomorphic tnorm for $X$ with respect to $\phi$.

Definition 16. ${ }^{29}$ Given a s-norm $Y$ and an automorphism $\phi$ on $[0,1]$, then the binary operation

$$
Y_{\phi}(p, q)=\phi^{-1}(Y(\phi(p), \phi(q)))
$$

is also a s-norm. $Y_{\phi}$ can be called as an isomorphic s-norm of $Y$ with respect to $\phi$.

\section{Frank operations for IVPFSs}

In this section, based on the Frank dual triple $\left(X_{F}, Y_{F}, \eta_{I}\right)$ and a special automorphism $\wp$ on $[0,1]$, we propose the concept of isomorphic Frank dual triple $\left(X_{F, \wp}, Y_{F, \wp}, \eta_{P}\right)$. Then, we define the Frank operations for IVPFSs in the use of the proposed triple. 


\subsection{Isomorphic Frank dual triple}

The operations for IVPFSs, which are based on the triple $\left(X_{F}, Y_{F}, \eta_{I}\right)$, do not satisfy the property of closure as can be demonstrated through the following example:

Example 1. Let $\widetilde{P}_{1}=([0.5,0.6],[0.7,0.8])$ and $\widetilde{P}_{2}=([0.6,0.8],[0.5,0.6])$ be two IVPFNs. If we define the addition operational law of IVPFNs based on the Frank dual triple $\left(X_{F}, Y_{F}, \eta_{I}\right)$, then we have

$$
\begin{array}{r}
\widetilde{P}_{1} \oplus_{F} \widetilde{P}_{2}=\left(\left[Y_{F}\left(p_{1}^{-}, p_{2}^{-}\right), Y_{F}\left(p_{1}^{+}, p_{2}^{+}\right)\right],\right. \\
\left.\left[X_{F}\left(q_{1}^{-}, q_{2}^{-}\right), X_{F}\left(q_{1}^{+}, q_{2}^{+}\right)\right]\right)
\end{array}
$$

If we let $\chi=1$, then

$$
\widetilde{P}_{1} \oplus_{F} \widetilde{P}_{2}=([0.80,0.92],[0.35,0.48]) .
$$

Evidently, $(0.92)^{2}+(0.48)^{2}>1$, which implies that $\widetilde{P}_{1} \oplus_{F} \widetilde{P}_{2}$ is not an IVPFN.

Example 1 demonstrates that the preceding operational laws is not closed for some special IVPFNs. Next, we devise a special automorphism on the unit interval $[0,1]$, which is rigid adherence to the relationship between IFSs and PFSs to be revealed.

Let $I=\left(\mu_{I}, v_{I}\right)$ and $P=\left(\mu_{P}, v_{P}\right)$ be an IFN and a PFN, respectively. From Definitions 1 and 2 , we have $\mu_{I}+v_{I} \leqslant 1$ and $\left(\mu_{P}\right)^{2}+\left(v_{P}\right)^{2} \leqslant$ 1. By using the standard negation $\eta_{I}$ and the Pythagorean negation $\eta_{P}$, two inequalities can be replaced by $\mu_{I} \leqslant \eta_{I}\left(v_{I}\right)$ and $\mu_{P} \leqslant \eta_{P}\left(v_{P}\right)$, respectively. Furthermore, through Theorem 1, we obtain another alternative form of these inequalities as follows: $\mu_{I} \leqslant \wp\left(\eta_{P}\left(\wp^{-1}\left(v_{I}\right)\right)\right)$ and $\mu_{P} \leqslant$ $\wp^{-1}\left(\eta_{I}\left(\wp\left(v_{P}\right)\right)\right)$, where $\wp(x)=x^{2}$ is an automorphism on $[0,1]$.

From the aforementioned results, it is clear that the constraint for PFNs can be expressed by the automorphism $\wp$ and the standard negation $\eta_{I}$. On the basis of Definitions 15 and 16, we develop an isomorphic Frank t-norm and an isomorphic Frank snorm with the application of the automorphism $\wp$.

Definition 17. Given the Frank t-norm $X_{F}$ and an automorphism $\wp(x)=x^{2}$ on the unit interval, then a binary operation $X_{F, \wp}$ on the unit interval satisfies the following condition:

$$
X_{F, \wp}(p, q)=\wp^{-1}\left(X_{F}(\wp(p), \wp(q))\right)
$$

is called an isomorphic Frank s-norm of $X_{F}$ with respect to $\wp$.

Theorem 4. Given the isomorphic Frank t-norm $X_{F, \wp}$, then

$$
\begin{aligned}
X_{F, \wp}(p, q) & =\hbar_{F, \wp}^{(-1)}\left(\hbar_{F, \wp}(p)+\hbar_{F, \wp}(q)\right) \\
& =\sqrt{\frac{\ln \left(1+\left(\chi^{p^{2}}-1\right)\left(\chi^{q^{2}}-1\right) /(\chi-1)\right)}{\ln \chi}}
\end{aligned}
$$

where $\hbar_{F, \wp}$ is the decreasing generator of $X_{F, \wp}$, $\hbar_{F, \wp}=\hbar_{F} \circ \wp$, and $\hbar_{F}$ is the decreasing generator of $X_{F}$.

Definition 18. Given the Frank s-norm $Y_{F}$ and an automorphism $\wp(x)=x^{2}$ on unit interval, if the operations $Y_{F, \wp}:[0,1]^{2} \rightarrow[0,1]$ satisfies

$$
Y_{F, \wp}(p, q)=\wp^{-1}\left(Y_{F}(\wp(p), \wp(q))\right) \text {, }
$$

then it can be called as an isomorphic Frank s-norm of $Y_{F}$ with respect to $\wp$.

Theorem 5. Given the isomorphic Frank s-norm $Y_{F, \wp}$, then

$$
\begin{aligned}
& Y_{F, \wp}(p, q)=g_{F, \wp}^{(-1)}\left(g_{F, \wp}(p)+g_{F, \wp}(q)\right) \\
& =\sqrt{1-\frac{\ln \left(1+\left(\chi^{1-p^{2}}-1\right)\left(\chi^{1-q^{2}}-1\right) /(\chi-1)\right)}{\ln \chi}}
\end{aligned}
$$

where $g_{F, \wp}$ is the decreasing generator of $Y_{F, \wp}$ such that $g_{F, \wp}=g_{F} \circ \wp$, and the $g_{F}$ is the decreasing generator of Frank s-norm $Y_{F}$.

Theorem 6. The triple $\left(X_{F, \wp}, Y_{F, \wp}, \eta_{P}\right)$ is also a dual triple, which is called an isomorphic Frank dual triple.

Proof. See Appendix A.

Then, the present isomorphic Frank triple is applied to define the interval-valued Pythagorean Frank operations.

Definition 19. Let $\widetilde{P}_{l}=\left(\left[p_{l}^{-}, p_{l}^{+}\right],\left[q_{l}^{-}, q_{l}^{+}\right]\right)(l=1,2)$ be two IVPFNs and $\chi \in(1, \infty)$. Then, the operational rules based on Frank isomorphic dual triple are defined as follows: 
(1) $\widetilde{P}_{1} \oplus_{F I} \widetilde{P}_{2}=\left(\left[Y_{F, \wp}\left(p_{1}^{-}, p_{2}^{-}\right), Y_{F, \wp}\left(p_{1}^{+}, p_{2}^{+}\right)\right],\left[X_{F, \wp}\left(q_{1}^{-}, q_{2}^{-}\right), X_{F, \wp}\left(q_{1}^{+}, q_{2}^{+}\right)\right]\right)$

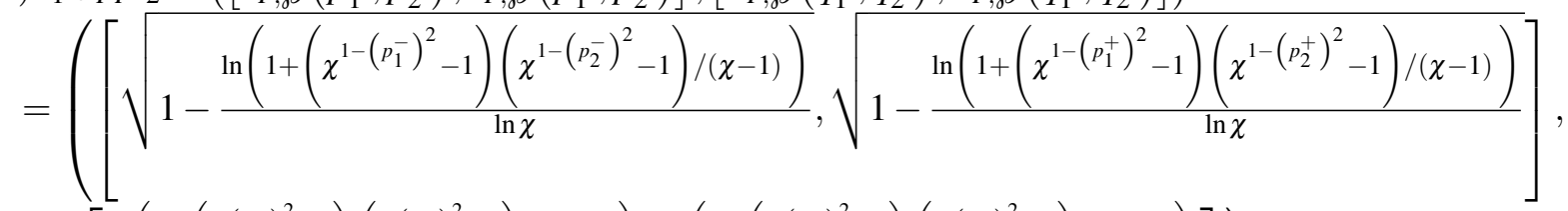

$\left.\left[\frac{\ln \left(1+\left(\chi^{\left(q_{1}^{-}\right)^{2}}-1\right)\left(\chi^{\left(q_{2}^{-}\right)^{2}}-1\right) /(\chi-1)\right)}{\ln \chi}, \frac{\ln \left(1+\left(\chi^{\left(q_{1}^{+}\right)^{2}}-1\right)\left(\chi^{\left(q_{2}^{+}\right)^{2}}-1\right) /(\chi-1)\right)}{\ln \chi}\right]\right)$

(2) $\widetilde{P}_{1} \otimes_{F I} \widetilde{P}_{2}=\left(\left[Y_{F, \wp}\left(p_{1}^{-}, p_{2}^{-}\right), Y_{F, \wp}\left(p_{1}^{+}, p_{2}^{+}\right)\right],\left[X_{F, \wp}\left(q_{1}^{-}, q_{2}^{-}\right), X_{F, \wp}\left(q_{1}^{+}, q_{2}^{+}\right)\right]\right)$

$\begin{aligned}= & \left(\left[\frac{\ln \left(1+\left(\chi^{\left(p_{1}^{-}\right)^{2}}-1\right)\left(\chi^{\left(p_{2}^{-}\right)^{2}}-1\right) /(\chi-1)\right)}{\ln \chi}, \frac{\ln \left(1+\left(\chi^{\left(p_{1}^{+}\right)^{2}}-1\right)\left(\chi^{\left(p_{2}^{+}\right)^{2}}-1\right) /(\chi-1)\right)}{\ln \chi}\right],\right. \\ & {\left.\left[\sqrt{1-\frac{\ln \left(1+\left(\chi^{1-\left(q_{1}^{-}\right)^{2}}-1\right)\left(\chi^{1-\left(q_{2}^{-}\right)^{2}}-1\right) /(\chi-1)\right.}{\ln \chi}}, \sqrt{1-\frac{\ln \left(1+\left(\chi^{1-\left(q_{1}^{+}\right)^{2}}-1\right)\left(\chi^{1-\left(q_{2}^{+}\right)^{2}}-1\right) /(\chi-1)\right)}{\ln \chi}}\right]\right) }\end{aligned}$

(3) $\kappa \widetilde{P}_{1}=\left(\left[g_{F, \wp}^{(-1)}\left(\kappa g_{F, \wp}\left(p_{1}^{-}\right)\right), g_{F, \wp}^{(-1)}\left(\kappa g_{F, \wp}\left(p_{1}^{+}\right)\right)\right],\left[\hbar_{F, \wp}^{(-1)}\left(\kappa \hbar_{F, \wp}\left(q_{1}^{-}\right)\right), \hbar_{F, \wp}^{(-1)}\left(\kappa \hbar_{F, \wp}\left(q_{1}^{+}\right)\right)\right]\right)$

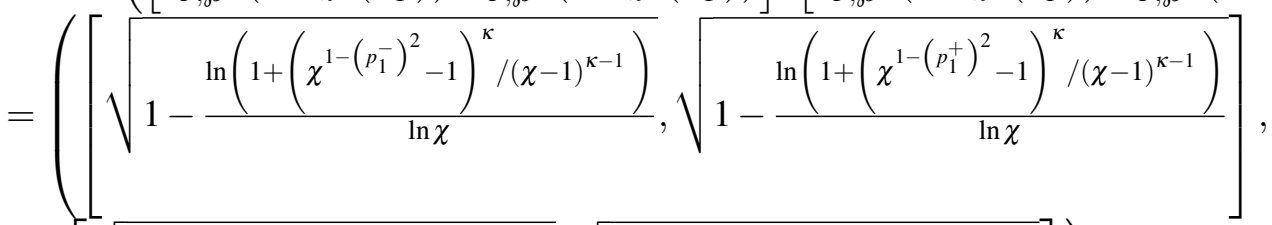

$\sqrt{\frac{\ln \left(1+\left(\chi^{\left(q_{1}^{-}\right)^{2}}-1\right)^{\kappa} /(\chi-1)^{\kappa-1}\right)}{\ln \chi}}, \sqrt{\left.\frac{\ln \left(1+\left(\chi^{\left(q_{1}^{+}\right)^{2}}-1\right)^{\kappa} /(\chi-1)^{\kappa-1}\right)}{\ln \chi}\right]}$

(4) $\widetilde{P}_{1}^{\kappa}=\left(\left[\hbar_{F, \wp}^{(-1)}\left(\kappa \hbar_{F, \wp}\left(p_{1}^{-}\right)\right), \hbar_{F, \wp}^{(-1)}\left(\kappa \hbar_{F, \wp}\left(p_{1}^{+}\right)\right)\right],\left[g_{F, \wp}^{(-1)}\left(\kappa g_{F, \wp}\left(q_{1}^{-}\right)\right), g_{F, \wp}^{(-1)}\left(\kappa g_{F, \wp}\left(q_{1}^{+}\right)\right)\right]\right)$

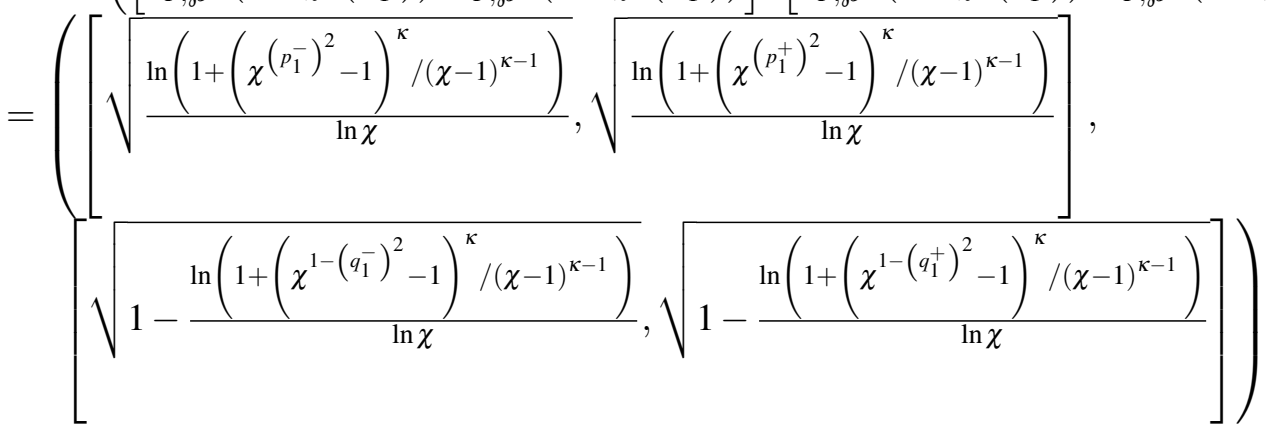

Theorem 7. The operations in Definition 19 are closed.

Proof. See Appendix B.

Theorem 8. Given two IVPFNS $\widetilde{P}_{l}=$ $\left(\left[p_{l}^{-}, p_{l}^{+}\right],\left[q_{l}^{-}, q_{l}^{+}\right]\right)(l=1,2)$, and $\kappa_{1}, \kappa_{2}, \kappa>0$, then

(1) $\widetilde{P}_{1} \oplus_{F I} \widetilde{P}_{2}=\widetilde{P}_{2} \oplus_{F I} \widetilde{P}_{1} . \quad$ (2) $\widetilde{P}_{1} \otimes_{F I} \widetilde{P}_{2}=\widetilde{P}_{2} \otimes_{F I} \widetilde{P}_{1}$.

(3) $\widetilde{P}_{1}^{\kappa} \oplus_{F I} \widetilde{P}_{2}^{\kappa}=\left(\widetilde{P}_{1} \otimes_{F I} \widetilde{P}_{2}\right)^{\kappa}$. 
(4) $\widetilde{P}^{\kappa_{1}} \otimes_{F I} \widetilde{P}^{\kappa_{2}}=\widetilde{P}^{\kappa_{1}+\kappa_{2}}$.

(5) $\kappa \widetilde{P}_{1} \oplus_{F I} \kappa \widetilde{P}_{2}=\kappa\left(\widetilde{P}_{1} \oplus_{F I} \widetilde{P}_{2}\right)$.

(6) $\kappa_{1} \widetilde{P} \oplus_{F I} \kappa_{2} \widetilde{P}=\left(\kappa_{1}+\kappa_{2}\right) \widetilde{P}$.

\section{IVPFFP operators}

In this section, the IVPFFPWA and IVPFFPWG operators are developed on the basis of the Pythagorean fuzzy Frank operations. In addition, their limiting cases are discussed.

\subsection{PA and PG operators}

The PA operator was originally developed by Yager $^{23}$. The desirable characteristic of the PA operator is its weighting vector that depends on the support function adopted among the aggregates. First, we review the concept of PA operator.

Definition 20. ${ }^{26}$ A function $P A: R^{n} \rightarrow R$ that satisfies

$$
P A\left(p_{1}, p_{2}, \cdots, p_{n}\right)=\sum_{l=1}^{n} \frac{\left(1+\Gamma\left(p_{l}\right)\right)}{\sum_{k=1}^{n}\left(1+\Gamma\left(p_{k}\right)\right)} p_{l}
$$

is called the power average (PA) operator, where $\Gamma\left(p_{l}\right)=\sum_{l \neq k}^{n} \Delta\left(p_{l}, p_{k}\right), \Delta\left(p_{l}, p_{k}\right)$ is the support from $p_{l}$ to $p_{k}$, and vice versa, which satisfies the following conditions:

(i) $0 \leqslant \Delta\left(p_{l}, p_{k}\right) \leqslant 1$. (ii) $\Delta\left(p_{l}, p_{k}\right)=\Delta\left(p_{k}, p_{l}\right)$.

(iii) If $\left|p_{l}-p_{k}\right|<\left|p_{l}-p_{j}\right|$, then $\Delta\left(p_{l}, p_{k}\right) \geqslant$ $\Delta\left(p_{l}, p_{j}\right)$.

The input arguments usually come from different sources with different degrees of importance. Thus, each argument should be assigned with a weight as a reflection of diverse significance. The power weighted average (PWA) operator is defined as follows.

Definition 21. A function $P W A: R^{n} \rightarrow R$ that satisfies

$P W A\left(p_{1}, p_{2}, \cdots, p_{n}\right)=\sum_{l=1}^{n} \frac{\omega_{l}\left(1+\Gamma\left(p_{l}\right)\right)}{\sum_{k=1}^{n} \omega_{k}\left(1+\Gamma\left(p_{k}\right)\right)} p_{l}$

is called the PWA, and $\omega_{l}(l \in N)$ is the weighting vector of $p_{l}(l \in N)$.
Theorem 9. If $\Delta\left(p_{l}, p_{k}\right)=a$ for all $l, k(l \neq k)$, then the PWA operator reduces to the WA operator:

$$
P W A\left(p_{1}, p_{2}, \cdots, p_{n}\right)=\sum_{l=1}^{n} \omega_{l} p_{l} .
$$

Motivated by the PA operator, the PG operator was further developed by $\mathrm{Xu}$ and Yager ${ }^{27}$.

Definition 22. ${ }^{27}$ A function $P G: R^{n} \rightarrow R$ that satisfies

$$
P G\left(p_{1}, p_{2}, \cdots, p_{n}\right)=\prod_{l=1}^{n} p_{l}^{\frac{\left(1+\Gamma\left(p_{l}\right)\right)}{\sum_{k=1}^{n}\left(1+\Gamma\left(p_{k}\right)\right)}},
$$

is called the called the PG operator.

Definition 23. A function $P W G: R^{n} \rightarrow R$ that satisfies

$$
P W G\left(p_{1}, p_{2}, \cdots, p_{n}\right)=\prod_{l=1}^{n} p_{l}^{\frac{\omega_{l}\left(1+\Gamma\left(p_{l}\right)\right)}{\sum_{k=1}^{n} \omega_{k}\left(1+\Gamma\left(p_{k}\right)\right)}} .
$$

is called the power weighted geometric (PWG) operator.

$$
\begin{aligned}
& \text { If } \omega_{l}=\frac{1}{n}(l \in N) \text {, then } \\
& P W G\left(p_{1}, p_{2}, \cdots, p_{n}\right)=P G\left(p_{1}, p_{2}, \cdots, p_{n}\right) .
\end{aligned}
$$

Theorem 10. If $\Delta\left(p_{l}, p_{k}\right)=a$ for all $l, k(l \neq k)$, then the PWG operator reduces to the WG operator:

$$
P W G\left(p_{1}, p_{2}, \cdots, p_{n}\right)=\prod_{l=1}^{n} p_{l}^{\omega_{l}} .
$$

From Definitions 21 and 23, it is evident that the weight $\frac{\omega_{l}\left(1+\Gamma\left(p_{l}\right)\right)}{\sum_{k=1}^{n} \omega_{k}\left(1+\Gamma\left(p_{k}\right)\right)}$ used for aggregation consists of $w_{l}$ and $\Gamma\left(p_{l}\right)(l \in N)$. If we let $u_{l}=$ $\frac{\omega_{l}\left(1+\Gamma\left(p_{l}\right)\right)}{\sum_{k=1}^{n} \omega_{k}\left(1+\Gamma\left(p_{k}\right)\right)}(l \in N)$, then we can easily obtain the aggregation weight $u_{l}$ increases with the increase of $w_{l}$ and $T\left(p_{l}\right)(l \in N)$.

\subsection{IVPFFPWA and IVPFFPWG operators}

Based on the PWA operator and the PWG operator, this section follows strictly the Frank operational laws to develop the IVPFFPWA and IVPFFPWG operators, and some properties of these operators are investigated. 


\subsubsection{IVPFFPWA operator}

According to the operational rules (1) and (3) in Definition 19 , the IVPFFPWA operator is provided as follows:

Definition 24. Given $n$ IVPFNs $\widetilde{P}_{l}=$ $\left(\left[p_{l}^{-}, p_{l}^{+}\right],\left[q_{l}^{-}, q_{l}^{+}\right]\right)(l \in N)$. The weights of $\widetilde{P}_{l}(l \in N)$ are $\omega_{l}(l \in N)$. The IVPFFPWA operator is given as

$$
\operatorname{IVPFFPWA}(\widetilde{\mathbb{P}})=\bigoplus_{l=1}^{n} \frac{\omega_{l}\left(1+\Gamma\left(\widetilde{P}_{l}\right)\right)}{\sum_{j=1}^{n} \omega_{j}\left(1+\Gamma\left(\widetilde{P}_{j}\right)\right)} \widetilde{P}_{l},
$$

where $\Gamma\left(\widetilde{P}_{l}\right)=\sum_{j \neq l}^{n} \Delta\left(\widetilde{P}_{l}, \widetilde{P}_{j}\right)$, and $\Delta\left(\widetilde{P}_{l}, \widetilde{P}_{j}\right)$ is the support from $\widetilde{P}_{l}$ to $\widetilde{P}_{j}$ and vice versa, which satisfies the following conditions:

(i) $0 \leqslant\left(\widetilde{P}_{l}, \widetilde{P}_{j}\right) \leqslant 1 . \quad$ (ii) $\Delta\left(\widetilde{P}_{l}, \widetilde{P}_{j}\right)=\Delta\left(\widetilde{P}_{j}, \widetilde{P}_{l}\right)$. (iii) $d\left(\widetilde{P}_{l}, \widetilde{P}_{j}\right)<d\left(\widetilde{P}_{l}, \widetilde{P}_{k}\right)$ implies $\Delta\left(\widetilde{P}_{l}, \widetilde{P}_{j}\right) \geqslant$ $\Delta\left(\widetilde{P}_{l}, \widetilde{P}_{k}\right) \cdot d\left(\widetilde{P}_{l}, \widetilde{P}_{j}\right)$ is defined in Definition 6.

Theorem 11. Given $n$ IVPFNS $\widetilde{P}_{l}=$ $\left(\left[p_{l}^{-}, p_{l}^{+}\right],\left[q_{l}^{-}, q_{l}^{+}\right]\right)(l \in N)$. Then

$$
\operatorname{IVPFFPWA}(\widetilde{\mathbb{P}})=\left(\left[P^{-}, P^{+}\right],\left[Q^{-}, Q^{+}\right]\right),
$$

and IVPFFPWA $(\widetilde{\mathbb{P}})$ is also an IVPFN, where

$$
\begin{aligned}
& P^{-}=g_{F, \wp}^{(-1)}\left(\sum_{l=1}^{n} u_{l} g_{F, \wp}\left(p_{l}^{-}\right)\right), \\
& P^{+}=g_{F, \wp}^{(-1)}\left(\sum_{l=1}^{n} u_{l} g_{F, \wp}\left(p_{l}^{+}\right)\right), \\
& Q^{-}=\hbar_{F, \wp}^{(-1)}\left(\sum_{l=1}^{n} u_{l} \hbar_{F, \wp}\left(q_{l}^{-}\right)\right), \\
& Q^{+}=\hbar_{F, \wp}^{(-1)}\left(\sum_{l=1}^{n} u_{l} \hbar_{F, \wp}\left(q_{l}^{+}\right)\right),
\end{aligned}
$$

Proof. See Appendix C.

Theorem 12. Given $n$ IVPFNs $\widetilde{P}_{l}=$ $\left(\left[p_{l}^{-}, p_{l}^{+}\right],\left[q_{l}^{-}, q_{l}^{+}\right]\right)(l \in N)$, then

$$
\begin{aligned}
& \operatorname{IVPFFPWA}(\widetilde{\mathbb{P}})=\left(\left[P^{-}, P^{+}\right],\left[Q^{-}, Q^{+}\right]\right) \\
& =\left(\left[\sqrt{1-\ln \left(1+\prod_{l=1}^{n}\left(\chi^{1-\left(p_{l}^{-}\right)^{2}}-1\right)^{u_{l}}\right) / \ln \chi}, \sqrt{1-\ln \left(1+\prod_{l=1}^{n}\left(\chi^{1-\left(p_{l}^{+}\right)^{2}}-1\right)^{u_{l}}\right) / \ln \chi}\right],\right. \\
& \left.\left[\sqrt{\ln \left(1+\prod_{l=1}^{n}\left(\chi^{\left(q_{l}^{-}\right)^{2}}-1\right)^{u_{l}}\right) / \ln \chi}, \sqrt{\ln \left(1+\prod_{l=1}^{n}\left(\chi^{\left(q_{l}^{+}\right)^{2}}-1\right)^{u_{l}}\right) / \ln \chi}\right]\right)
\end{aligned}
$$

where $u_{l}=\frac{\omega_{l}\left(1+\Gamma\left(\widetilde{P}_{l}\right)\right)}{\sum_{j=1}^{n} \omega_{j}\left(1+\Gamma\left(\widetilde{P}_{j}\right)\right)}(l \in N)$.

Proof. See Appendix D.

Theorem 13. (1) If $\omega_{l}=1 / n(l \in N)$, then $u_{l}=$ $\frac{\left(1+\Gamma\left(\widetilde{P}_{l}\right)\right)}{\sum_{j=1}^{n}\left(1+\Gamma\left(\widetilde{P}_{j}\right)\right)}(l \in N)$.Therefore, the IVPFFPWA operator reduces to the IVPFFPA operator.

(2) If $\Delta\left(\widetilde{P}_{l}, \widetilde{P}_{j}\right)=a$ for all $l, j(l \neq j)$, then $u_{l}=$ $\omega_{l}(l \in N)$. Therefore, the IVPFFPWA operator reduces to the IVPFFWA operator.
Theorem 14. Given $n$ IVPFNs $\widetilde{P}_{l}=$ $\left(\left[p_{l}^{-}, p_{l}^{+}\right],\left[q_{l}^{-}, q_{l}^{+}\right]\right)(l \in N)$, the following properties hold.

(i) Commutativity: If $\widetilde{P}_{l}(l \in N)$ is any permutation of $\widetilde{P}_{l}(l \in N)$, then

$$
\operatorname{IVPFFPWA}(\widetilde{\mathbb{P}})=\operatorname{IVPFFPWA}\left(\widetilde{P}_{1}, \widetilde{P}_{2}^{\prime}, \cdots, \widetilde{P}_{n}\right) .
$$

(ii) Idempotency: If $\widetilde{P}_{l}=\widetilde{P}=\left(\left[p^{-}, p^{+}\right],\left[q^{-}, q^{+}\right]\right)(l \in N)$ , then

$$
\operatorname{IVPFFPWA}(\widetilde{\mathbb{P}})=\widetilde{P}
$$


(iii) Boundedness: If

Definition 25. Given $n$ IVPFNs $\widetilde{P}_{l}=$ $\left.\widetilde{P^{-}}\right\}^{\left(\left[p_{l}^{-}, p_{l}^{+}\right],\left[q_{l}^{-}, q_{l}^{+}\right]\right)(l \in N) \text {. The IVPFFPWG op- }}$ $\widetilde{P}_{\min }=\left(\left[\min _{l}\left\{p_{l}^{-}\right\}, \min _{l}\left\{p_{l}^{+}\right\}\right],\left[\max _{l}\left\{q_{l}^{-}\right\}, \max _{l}\left\{q_{l}^{+}\right\}\right]\right.$e)ator is given as

$\widetilde{P}_{\max }=\left(\left[\max _{l}\left\{p_{l}^{-}\right\}, \max _{l}\left\{p_{l}^{+}\right\}\right],\left[\min _{l}\left\{q_{l}^{-}\right\}, \min _{l}\left\{q_{l}^{+}\right\}\right]\right)$,

then

$$
\widetilde{P}_{\min } \leqslant \operatorname{IVPFFPWA}(\widetilde{\mathbb{P}}) \leqslant \widetilde{P}_{\max }
$$

(iv) Monotonicity: Let $\widetilde{Q}_{l}=\left(\left[m_{l}^{-}, m_{l}^{+}\right],\left[n_{l}^{-}, n_{l}^{+}\right]\right)(l \in N)$ be a family of IVPFNs. If $\widetilde{Q}_{l} \leqslant \widetilde{P}_{l}(l \in N)$, and $\Delta\left(\widetilde{P}_{l}, \widetilde{P}_{j}\right)=\Delta\left(\widetilde{Q}_{l}, \widetilde{Q}_{j}\right)$ for all $l, j(l \neq j)$, then

$$
\operatorname{IVPFFPWA}(\widetilde{\mathbb{Q}}) \leqslant \operatorname{IVPFFPWA}(\widetilde{\mathbb{P}}) .
$$

Proof. See Appendix E.

where $u_{l}=\frac{\omega_{l}\left(1+\Gamma\left(\widetilde{P}_{l}\right)\right)}{\sum_{j=1}^{n} \omega_{j}\left(1+\Gamma\left(\widetilde{P}_{j}\right)\right)}(l \in N)$.

$$
\operatorname{IVPFFPWG}(\widetilde{\mathbb{P}})=\stackrel{\otimes}{l=1}_{l}^{u_{l}^{u_{l}}},
$$

Theorem 15. Given $n$ IVPFNs $\widetilde{P}_{l}=$ $\left(\left[p_{l}^{-}, p_{l}^{+}\right],\left[q_{l}^{-}, q_{l}^{+}\right]\right)(l \in N)$. Then

$\operatorname{IVPFFPWG}(\widetilde{\mathbb{P}})$

$$
\begin{aligned}
& =\left(\left[\hbar_{F, \wp}^{(-1)}\left(\sum_{l=1}^{n} u_{l} \hbar_{F, \wp}\left(p_{l}^{-}\right)\right), \hbar_{F, \wp}^{(-1)}\left(\sum_{l=1}^{n} u_{l} \hbar_{F, \wp}\left(p_{l}^{+}\right)\right)\right],\right. \\
& \left.\left[g_{F, \wp}^{(-1)}\left(\sum_{l=1}^{n} u_{l} g_{F, \wp}\left(q_{l}^{-}\right)\right), g_{F, \wp}^{(-1)}\left(\sum_{l=1}^{n} u_{l} g_{F, \wp}\left(q_{l}^{+}\right)\right)\right]\right)
\end{aligned}
$$

\subsubsection{IVPFFPWG operator}

According to the operational rules (2) and (4) in Definition 19, the IVPFFPWG operator can be defined as follows:

and IVPFFPWG $\left(\widetilde{P}_{1}, \widetilde{P}_{2}, \cdots, \widetilde{P}_{n}\right)$ is also an IVPFN.

Theorem 16. Given $n$ IVPFNs $\widetilde{P}_{l}=$ $\left(\left[p_{l}^{-}, p_{l}^{+}\right],\left[q_{l}^{-}, q_{l}^{+}\right]\right)(l \in N)$, then

$$
\begin{aligned}
\operatorname{IVPFFPWG}(\widetilde{\mathbb{P}})= & \left(\left[\sqrt{\ln \left(1+\prod_{l=1}^{n}\left(\chi^{\left(p_{l}^{-}\right)^{2}}-1\right)^{u_{l}}\right) / \ln \chi}, \sqrt{\ln \left(1+\prod_{l=1}^{n}\left(\chi^{\left(p_{l}^{+}\right)^{2}}-1\right)^{u_{l}}\right) / \ln \chi}\right],\right. \\
& {\left[\sqrt{1-\ln \left(1+\prod_{l=1}^{n}\left(\chi^{1-\left(q_{l}^{-}\right)^{2}}-1\right)^{u_{l}}\right) / \ln \chi}, \sqrt{1-\ln \left(1+\prod_{l=1}^{n}\left(\chi^{\left.\left.1-\left(q_{l}^{+}\right)^{2}-1\right)^{u_{l}}\right) / \ln \chi}\right]\right)}\right.}
\end{aligned}
$$

Theorem 17. (1) If $\omega_{l}=1 / n(l \in N)$, then $u_{l}=$ $\frac{\left(1+\Gamma\left(\widetilde{P}_{l}\right)\right)}{\sum_{j=1}^{n}\left(1+\Gamma\left(\widetilde{P}_{j}\right)\right)}(l \in N)$. Therefore, the IVPFFPWG operator reduces to the IVPFFPG operator.

(2) If $\Delta\left(\widetilde{P}_{l}, \widetilde{P}_{j}\right)=a$ for all $l, j(l \neq j)$, then $u_{l}=$ $\omega_{l}(l \in N)$. Therefore, the IVPFFPWG operator reduces to the IVPFFWG operator.

Theorem 18. Given $n$ IVPFNs $\widetilde{P}_{l}=$ $\left(\left[p_{l}^{-}, p_{l}^{+}\right],\left[q_{l}^{-}, q_{l}^{+}\right]\right)(l \in N)$, the following properties hold.

(i) Commutativity: If $\widetilde{P}_{l}^{\prime}(l \in N)$ is any permutation of $\widetilde{P}_{l}(l \in N)$, then

$\operatorname{IVPFFPWG}(\widetilde{\mathbb{P}})=\operatorname{IVPFFPWG}\left(\widetilde{P}_{1}, \widetilde{P}_{2}, \cdots, \widetilde{P}_{n}\right)$.

(ii) Idempotency: If $\widetilde{P}_{l}=\widetilde{P}=\left(\left[p^{-}, p^{+}\right],\left[q^{-}, q^{+}\right]\right)(l \in N)$ , then

$$
\operatorname{IVPFFPWG}(\widetilde{\mathbb{P}})=\widetilde{P} .
$$

(iii) Boundedness: Let $\widetilde{P}_{\min }$ and $\widetilde{P}_{\min }$ be the IVPFNs in Theorem 14, then

$$
\widetilde{P}_{\min } \leqslant \operatorname{IVPFFPWG}(\widetilde{\mathbb{P}}) \leqslant \widetilde{P}_{\max } .
$$


(iv) Monotonicity: Let $\widetilde{Q}_{l}=\left(\left[m_{l}^{-}, m_{l}^{+}\right],\left[n_{l}^{-}, n_{l}^{+}\right]\right)(l \in N)$ be a family of IVPFNs. If $\widetilde{Q}_{l} \leqslant \widetilde{P}_{l}(l \in N)$, and $\Delta\left(\widetilde{P}_{l}, \widetilde{P}_{j}\right)=\Delta\left(\widetilde{Q}_{l}, \widetilde{Q}_{j}\right)$ for all $l, j(l \neq j)$, then

$$
\operatorname{IVPFFPWG}(\widetilde{\mathbb{Q}}) \leqslant \operatorname{IVPFFPWG}(\widetilde{\mathbb{P}}) \text {. }
$$

Theorem 19. Given $n$ IVPFNS $\quad \widetilde{P}_{l}=$ $\left(\left[p_{l}^{-}, p_{l}^{+}\right],\left[q_{l}^{-}, q_{l}^{+}\right]\right)(l \in N)$, then (1) IVPFFPWG $(\widetilde{\mathbb{P}})=\left(\operatorname{IVPFFPWA}\left(\widetilde{P}_{1}^{c}, \widetilde{P}_{2}^{c}, \cdots, \widetilde{P}_{n}^{c}\right)\right)^{c}$ (2) IVPFFPWA $(\widetilde{\mathbb{P}})=\left(\operatorname{IVPFFPWG}\left(\widetilde{P}_{1}^{c}, \widetilde{P}_{2}^{c}, \cdots, \widetilde{P}_{n}^{c}\right)\right)^{c}$

Proof. We only prove (1). By Definition $5, \widetilde{P}_{l}^{c}=$ $\left(\left[q_{l}^{-}, q_{l}^{+}\right],\left[p_{l}^{-}, p_{l}^{+}\right]\right)(l \in N)$. Then, from Theorem 7 , we have

$$
\begin{aligned}
& \left(\operatorname{IVPFFPWA}\left(\widetilde{P}_{1}^{c}, \widetilde{P}_{2}^{c}, \cdots, \widetilde{P}_{n}^{c}\right)\right)^{c} \\
& =\left(\left[g_{F, \wp}^{(-1)}\left(\sum_{l=1}^{n} u_{l} g_{F, \wp}\left(q_{l}^{-}\right)\right), g_{F, \wp}^{(-1)}\left(\sum_{l=1}^{n} u_{l} g_{F, \wp}\left(q_{l}^{+}\right)\right)\right]\right. \\
& \left.\left[\hbar_{F, \wp}^{(-1)}\left(\sum_{l=1}^{n} u_{l} \hbar_{F, \wp}\left(p_{l}^{-}\right)\right), \hbar_{F, \wp}^{(-1)}\left(\sum_{l=1}^{n} u_{l} \hbar_{F, \wp}\left(p_{l}^{+}\right)\right)\right]\right)^{c} \\
& =\left(\left[\hbar_{F, \wp}^{(-1)}\left(\sum_{l=1}^{n} u_{l} \hbar_{F, \wp}\left(p_{l}^{-}\right)\right), \hbar_{F, \wp}^{(-1)}\left(\sum_{l=1}^{n} u_{l} \hbar_{F, \wp}\left(p_{l}^{+}\right)\right)\right],\right. \\
& \left.\left[g_{F, \wp}^{(-1)}\left(\sum_{l=1}^{n} u_{l} g_{F, \wp}\left(q_{l}^{-}\right)\right), g_{F, \wp}^{(-1)}\left(\sum_{l=1}^{n} u_{l} g_{F, \wp}\left(q_{l}^{+}\right)\right)\right]\right) \\
& =I V P F F P W G(\widetilde{\mathbb{P}})
\end{aligned}
$$

\subsection{Limiting cases of IVPFFP operators}

Before moving forward to the discussion of the limiting cases of the IVPFFPWA and IVPFFPWG operators, we introduce two special aggregation functions with adjustable parameters.

Definition 26. A function $A F_{\chi}:[0,1]^{n} \rightarrow[0,1]$ that satisfies

$A F_{\chi}\left(p_{1}, \cdots, p_{n}\right)=\sqrt{\ln \left(1+\prod_{l=1}^{n}\left(\chi^{p_{l}^{2}}-1\right)^{\omega_{l}}\right) / \ln \chi}$ is called Pythagorean Frank aggregation function, and where $\chi \in(1, \infty)$ and $W$ is the aggregation weighting vector.

Definition 27. A function $A F_{\chi}^{d}:[0,1]^{n} \rightarrow[0,1]$ that satisfies

$A F_{\chi}^{d}\left(p_{1}, \cdots, p_{n}\right)=\eta_{P}\left(A F_{\chi}\left(\eta_{P}\left(p_{1}\right), \cdots, \eta_{P}\left(p_{n}\right)\right)\right)$

$=\sqrt{1-\ln \left(1+\prod_{l=1}^{n}\left(\chi^{1-p_{l}^{2}}-1\right)^{\omega_{l}}\right) / \ln \chi}$

is called dual Pythagorean Frank aggregation function.

Theorem 20. Let $A F_{\chi}$ and $A F_{\chi}^{d}$ be the functions defined in Definitions 26 and 27, then

(1) $\lim _{\chi \rightarrow 1} A F_{\chi}\left(p_{1}, \cdots, p_{n}\right)=\prod_{l=1}^{n} p_{l}^{\omega_{l}}$;

(2) $\lim _{\chi \rightarrow 1} A F_{\chi}^{d}\left(p_{1}, \cdots, p_{n}\right)=\sqrt{1-\prod_{l=1}^{n}\left(1-p_{l}^{2}\right)^{\omega_{l}}}$.

(3) $\lim _{\chi \rightarrow \infty} A F_{\chi}\left(p_{1}, \cdots, p_{n}\right)=\sqrt{\sum_{l=1}^{n} \omega_{l} p_{l}^{2}}$;

(4) $\lim _{\chi \rightarrow \infty} A F_{\chi}^{d}\left(p_{1}, \cdots, p_{n}\right)=\sqrt{\sum_{l=1}^{n} \omega_{l} p_{l}^{2}}$.

Proof. See Appendix F.

Theorem 21. Given $n$ IVPFNs $\widetilde{P}_{l}=$ $\left(\left[p_{l}^{-}, p_{l}^{+}\right],\left[q_{l}^{-}, q_{l}^{+}\right]\right)(l \in N)$, then

(1) IVPFFPWA $(\widetilde{\mathbb{P}})$

$$
\begin{gathered}
=\left(\left[A F_{\chi}^{d}\left(p_{1}^{-}, \cdots, p_{n}^{-}\right), A F_{\chi}^{d}\left(p_{1}^{+}, \cdots, p_{n}^{+}\right)\right]\right. \\
\left.\left[A F_{\chi}\left(q_{1}^{-}, \cdots, q_{n}^{-}\right), A F_{\chi}\left(q_{1}^{+}, \cdots, q_{n}^{+}\right)\right]\right)
\end{gathered}
$$

(2) $\operatorname{IVPFFPWG}(\widetilde{\mathbb{P}})$

$$
\begin{gathered}
=\left(\left[A F_{\chi}\left(p_{1}^{-}, \cdots, p_{n}^{-}\right), A F_{\chi}\left(p_{1}^{+}, \cdots, p_{n}^{+}\right)\right]\right. \\
\left.\left[A F_{\chi}^{d}\left(q_{1}^{-}, \cdots, q_{n}^{-}\right), A F_{\chi}^{d}\left(q_{1}^{+}, \cdots, q_{n}^{+}\right)\right]\right)
\end{gathered}
$$

and where $u_{l}=\frac{\omega_{l}\left(1+\Gamma\left(\widetilde{P}_{l}\right)\right)}{\sum_{j=1}^{n} \omega_{j}\left(1+\Gamma\left(\widetilde{P}_{j}\right)\right)}(l \in N)$ is the aggregation weighting vector of $\widetilde{P}_{l}(l \in N)$.

Theorem 22. Given $n$ IVPFNs $\widetilde{P}_{l}=$ $\left(\left[p_{l}^{-}, p_{l}^{+}\right],\left[q_{l}^{-}, q_{l}^{+}\right]\right)(l \in N)$, and if $\chi \rightarrow 1$, then 
(1) the IVPFFPWA operator reduces to the IVPFPWA operator:

$\lim _{\chi \rightarrow 1} \operatorname{IVPFFPWA}(\widetilde{\mathbb{P}})=\operatorname{IVPFPWA}(\widetilde{\mathbb{P}})$

$=\left(\left[\sqrt{1-\prod_{l=1}^{n}\left(1-\left(p_{l}^{-}\right)^{2}\right)^{u_{l}}}, \sqrt{1-\prod_{l=1}^{n}\left(1-\left(p_{l}^{+}\right)^{2}\right)^{u_{l}}}\right]\right.$ $\left.\left[\prod_{l=1}^{n}\left(q_{l}^{-}\right)^{u_{l}}, \prod_{l=1}^{n}\left(q_{l}^{+}\right)^{u_{l}}\right]\right)$

(2) the IVPFFPWG operator reduces to the IVPF$P W G$ operator:

$\lim _{\chi \rightarrow 1} \operatorname{IVPFFPWG}(\widetilde{\mathbb{P}})=\operatorname{IVPFPWG}(\widetilde{\mathbb{P}})$

$=\left(\left[\prod_{l=1}^{n}\left(p_{l}^{-}\right)^{u_{l}}, \prod_{l=1}^{n}\left(p_{l}^{+}\right)^{u_{l}}\right]\right.$,

$\left.\left[\sqrt{1-\prod_{l=1}^{n}\left(1-\left(q_{l}^{-}\right)^{2}\right)^{u_{l}}}, \sqrt{1-\prod_{l=1}^{n}\left(1-\left(q_{l}^{+}\right)^{2}\right)^{u_{l}}}\right]\right)$

where $u_{l}=\frac{\omega_{l}\left(1+\Gamma\left(\widetilde{P}_{l}\right)\right)}{\sum_{j=1}^{n} \omega_{j}\left(1+\Gamma\left(\widetilde{P}_{j}\right)\right)}(l \in N)$.

Theorem 23. If $\Delta\left(\widetilde{P}_{l}, \widetilde{P}_{j}\right)=a$ for all $l, j(l \neq j)$, then $u_{l}=\omega_{l}(l \in N)$. Therefore,

(1) The IVPFPWA operator reduces to the IVPFWA operator:

$\lim _{\chi \rightarrow 1} I V P F P W A(\widetilde{\mathbb{P}})=I V P F W A(\widetilde{\mathbb{P}})$

$=\left(\left[\sqrt{1-\prod_{l=1}^{n}\left(1-\left(p_{l}^{-}\right)^{2}\right)^{\omega_{l}}}, \sqrt{1-\prod_{l=1}^{n}\left(1-\left(p_{l}^{+}\right)^{2}\right)^{\omega_{l}}}\right]^{o}\right.$,

$\left.\left[\prod_{l=1}^{n}\left(q_{l}^{-}\right)^{\omega_{l}}, \prod_{l=1}^{n}\left(q_{l}^{+}\right)^{\omega_{l}}\right]\right)$

This aggregation operator has been proposed in $^{12,13}$.

(2) Then, the IVPFPWG operator reduces to the IVPFWG operator:

$\lim _{\chi \rightarrow 1} \operatorname{IVPFFPWG}(\widetilde{\mathbb{P}})=I V P F W G(\widetilde{\mathbb{P}})$

$=\left(\left[\prod_{l=1}^{n}\left(p_{l}^{-}\right)^{\omega_{l}}, \prod_{l=1}^{n}\left(p_{l}^{+}\right)^{\omega_{l}}\right]\right.$,

$\left.\left[\sqrt{1-\prod_{l=1}^{n}\left(1-\left(q_{l}^{-}\right)^{2}\right)^{\omega_{l}}}, \sqrt{1-\prod_{l=1}^{n}\left(1-\left(q_{l}^{+}\right)^{2}\right)^{\omega_{l}}}\right]\right)$
This operator has been proposed $\mathrm{in}^{12,13}$.

Theorem 24. Given $n$ IVPFNs $\widetilde{P}_{l}=$ $\left(\left[p_{l}^{-}, p_{l}^{+}\right],\left[q_{l}^{-}, q_{l}^{+}\right]\right)(l \in N)$, and if $\chi \rightarrow \infty$, then

(1) The IVPFFPWA operator reduces to the intervalvalued Pythagorean fuzzy Frank power weighted quadratic (IVPFFPWQ) operator:

$$
\begin{aligned}
& \lim _{\chi \rightarrow \infty} \operatorname{IVPFFPWA}(\widetilde{\mathbb{P}})=\operatorname{IVPFFPWQ}(\widetilde{\mathbb{P}}) \\
& =\left(\left[\sqrt{\sum_{l=1}^{n} u_{l}\left(p_{l}^{-}\right)^{2}}, \sqrt{\sum_{l=1}^{n} u_{l}\left(p_{l}^{+}\right)^{2}}\right],\right. \\
& \left.\left[\sqrt{\sum_{l=1}^{n} u_{l}\left(q_{l}^{-}\right)^{2}}, \sqrt{\sum_{l=1}^{n} u_{l}\left(q_{l}^{+}\right)^{2}}\right]\right)
\end{aligned}
$$

(2) The IVPFFPWG operator reduce to the IVPFF$P W Q$ operator:

$$
\lim _{\chi \rightarrow \infty} \operatorname{IVPFFPWG}(\widetilde{\mathbb{P}})=I V P F F P W Q(\widetilde{\mathbb{P}})
$$

where $u_{l}=\frac{\omega_{l}\left(1+\Gamma\left(\widetilde{P}_{l}\right)\right)}{\sum_{j=1}^{n} \omega_{j}\left(1+\Gamma\left(\widetilde{P}_{j}\right)\right)}(l \in N)$.

Theorem 25. If $\Delta\left(\widetilde{P}_{l}, \widetilde{P}_{j}\right)=a$ for all $l, j(l \neq j)$, then $u_{l}=\omega_{l}(l \in N)$, and therefore the IVPFFPWQ operator reduces to IVPFWQ operator:

$$
\begin{aligned}
& \lim _{\chi \rightarrow \infty} \operatorname{IVPFFPWA}(\widetilde{\mathbb{P}})=\operatorname{IVPFPWQ}(\widetilde{\mathbb{P}}) \\
& =\left(\left[\sqrt{\sum_{l=1}^{n} \omega_{l}\left(p_{l}^{-}\right)^{2}}, \sqrt{\sum_{l=1}^{n} \omega_{l}\left(p_{l}^{+}\right)^{2}}\right],\right. \\
& \left.\left[\sqrt{\sum_{l=1}^{n} \omega_{l}\left(q_{l}^{-}\right)^{2}}, \sqrt{\sum_{l=1}^{n} \omega_{l}\left(q_{l}^{+}\right)^{2}}\right]\right)
\end{aligned}
$$

From the theorems mentioned, some limiting cases of IVPFFPWA and IVPFFPWG operators are summarized in Table 1 with different choices of parameter $\chi$ and aggregation weights $u_{l}$, where

$$
v_{l}=\left(1+\Gamma\left(\widetilde{P}_{l}\right)\right) / \sum_{j=1}^{n}\left(1+\Gamma\left(\widetilde{P}_{j}\right)\right)(l \in N) .
$$


Table 1: Special cases of proposed operators

\begin{tabular}{lclll}
\hline Operators & $u_{l}$ and $\chi$ & Reduced operator & $u_{l}$ & Reduced operator \\
\hline IVPFFPWA & $u_{l}=\omega_{l}$ & IVPFFWA(Theorem 13(2)) & & \\
IVPFFPWA & $u_{l}=v_{l}$ & IVPFFWA(Theorem 13(1)) & & \\
IVPFFPWA & $\chi \rightarrow 1$ & IVPFPWA(Theorem 22(1)) & $u_{l}=\omega_{l}$ & IVPFWA(Theorem 23(1)) \\
IVPFFPWA & $\chi \rightarrow \infty$ & IVPFPWQ(Theorem 24(1)) & $u_{l}=\omega_{l}$ & IVPFWQ(Theorem 25) \\
IVPFFPWG & $u_{l}=\omega_{l}$ & IVPFFWG(Theorem 17(2)) & & \\
IVPFFPWG & $u_{l}=v_{l}$ & IVPFFWG(Theorem 17(1)) & & \\
IVPFFPWG & $\chi \rightarrow 1$ & IVPFPWG(Theorem 22(2)) & $u_{l}=\omega_{l}$ & IVPFWG(Theorem 23(2)) \\
IVPFFPWG & $\chi \rightarrow \infty$ & IVPFPWQ(Theorem 24(2)) & $u_{l}=\omega_{l}$ & IVPFWQ(Theorem 25) \\
\hline
\end{tabular}

\section{A novel decision-making approach for MAGDM with IVPFNs}

Let sets of $m$ alternatives and $n$ attributes be $A L_{i}(i \in M)$ and $C_{j}(j \in N)$, respectively. Let $\omega_{j}(j \in N)$ be the weights for $C_{j}(j \in N)$. Let $d_{k}(k \in T)$ be a set of $t$ DMs, and their weights are $\lambda_{k}(k \in T)$. The alternatives $A L_{i}(i \in M)$ are assessed by the DMs with IVPFNs $\widetilde{P}_{i j}^{k}=$ $\left(\left[p_{i j}^{-k}, p_{i j}^{+k}\right],\left[q_{i j}^{-k}, q_{i j}^{+k}\right]\right)$ based on $C_{j}(j \in N)$. The decision matrixes are expressed as $D^{k}=$ $\left(\widetilde{P}_{i j}^{k}\right)_{m \times n}(k \in T)$. One method developed to obtain the best solution to this problem is based on the proposed Frank aggregation operators. The steps of the decision-making method are provided as follows:

Step 1. Following Definition 6, the supports between $\widetilde{P}_{i j}^{k}(k \in T)$ and $\widetilde{P}_{i j}^{l}(l \in T)$ can be calculated as:

$$
\Delta\left(\widetilde{P}_{i j}^{k}, \widetilde{P}_{i j}^{l}\right)=1-d\left(\widetilde{P}_{i j}^{k}, \widetilde{P}_{i j}^{l}\right)
$$

Step 2. Utilize the weights $\lambda_{k}(k \in T)$ of the DMs $d_{k}(k \in T)$ to obtain the support $\Gamma\left(\widetilde{P}_{i j}^{k}\right)(k \in T)$ of the IVPFN $\widetilde{P}_{i j}^{k}(k \in T)$ by the other IVPFNs $\widetilde{P}_{i j}^{l}(l \in T ; l \neq k)$ :

$\Gamma\left(\widetilde{P}_{i j}^{k}\right)=\sum_{l \neq k}^{t} \Delta\left(\widetilde{P}_{i j}^{k}, \widetilde{P}_{i j}^{l}\right)=\sum_{l \neq k}^{t}\left(1-d\left(\widetilde{P}_{i j}^{k}, \widetilde{P}_{i j}^{l}\right)\right)$,

and obtain the weights $\zeta_{i j}^{k}(k \in T)$ associated with the IVPFN $\widetilde{P}_{i j}^{k}(k \in T)$ :

$$
\zeta_{i j}^{k}=\frac{\lambda_{k}\left(1+T\left(\widetilde{\alpha}_{i j}^{k}\right)\right)}{\sum_{k=1}^{t} \lambda_{k}\left(1+T\left(\widetilde{\alpha}_{i j}^{k}\right)\right)} .
$$

Step 3.Use the IVPFFPWA operator (51) or the IVPFFPWG operator (52) to aggregate the decision matrixes $D^{k}=\left(\widetilde{P}_{i j}^{k}\right)_{m \times n}(k \in T)$ to the collective matrix $D=\left(\widetilde{P}_{i j}\right)_{m \times n}$, and $\widetilde{P}_{i j}=$ $\left(\left[p_{i j}^{-}, p_{i j}^{+}\right],\left[q_{i j}^{-}, q_{i j}^{+}\right]\right)$.

$$
\begin{gathered}
\widetilde{P}_{i j}=\operatorname{IVPFFPWA}\left(\widetilde{P}_{i j}^{1}, \widetilde{P}_{i j}^{2}, \cdots, \widetilde{P}_{i j}^{t}\right) \\
\widetilde{P}_{i j}=\operatorname{IVPFFPWG}\left(\widetilde{P}_{i j}^{1}, \widetilde{P}_{i j}^{2}, \cdots, \widetilde{P}_{i j}^{t}\right)
\end{gathered}
$$

Step 4. To obtain the comprehensive preference value $\widetilde{P}_{i}(i \in M)$ of the alternative $A L_{i}(i \in M)$, we fuse all the values $\widetilde{P}_{i j}(j \in N)$ in $D=\left(\widetilde{P}_{i j}\right)_{m \times n}$ by applying the IVPFFWA operator (53) or the IVPFFWG operator (54).

$$
\begin{aligned}
& \widetilde{P}_{i}=\operatorname{IVPFFWA}\left(\widetilde{P}_{i 1}, \widetilde{P}_{i 2}, \cdots, \widetilde{P}_{i n}\right) \\
& \widetilde{P}_{i}=\operatorname{IVPFFWG}\left(\widetilde{P}_{i 1}, \widetilde{P}_{i 2}, \cdots, \widetilde{P}_{i n}\right)
\end{aligned}
$$

Step 5. From Definition 4, obtain the score values $\operatorname{Sco}\left(\widetilde{P}_{i}\right)(i \in M)$. Then, we obtain the ranking of $\widetilde{P}_{i}(i \in M)$.

Step 6. According to the ranking of $\widetilde{P}_{i}(i \in M)$, we obtain the ranking of $A_{i}(i \in M)$.

$$
\begin{aligned}
& d\left(\widetilde{P}_{i j}^{k}, \widetilde{P}_{i j}^{l}\right) \\
& =\frac{1}{4}\left(\left|\left(p_{i j}^{-k}\right)^{2}-\left(p_{i j}^{-l}\right)^{2}\right|+\left|\left(p_{i j}^{+k}\right)^{2}-\left(p_{i j}^{+l}\right)^{2}\right|\right) \\
& +\frac{1}{4}\left(\left|\left(q_{i j}^{-k}\right)^{2}-\left(q_{i j}^{-l}\right)^{2}\right|+\left|\left(q_{i j}^{+k}\right)^{2}-\left(q_{i j}^{+l}\right)^{2}\right|\right) \\
& +\frac{1}{4}\left(\left|\left(\pi_{i j}^{-k}\right)^{2}-\left(\pi_{i j}^{-l}\right)^{2}\right|+\left|\left(\pi_{i j}^{+k}\right)^{2}-\left(\pi_{i j}^{+l}\right)^{2}\right|\right)
\end{aligned}
$$




\section{Numerical examples}

\subsection{A GDM problem of investment selection}

The background regarding an investment selection problem $\mathrm{in}^{30}$ is used to test the feasibility of the proposed decision-making approach in the previous section. In this problem, firstly, four essential attributes $C_{j}\left(j \in N_{4}\right)$ have to be analyzed, which are risk management, growth ability, social and political influence, and environmental protection strategy analysis. Considered here are four candidate alternatives $A_{i}\left(i \in M_{4}\right)$ : automotive enterprises, food enterprise, computer enterprise, and arms enterprise. The evaluation information is provided by the three experts $d_{k}\left(k \in T_{3}\right)$ on the four alternatives $A_{i}\left(i \in M_{4}\right)$ under $C_{j}\left(j \in N_{4}\right)$ in the manifestation of IVPFNs $\widetilde{P}_{i j}^{k}\left(i \in M_{4}, j \in N_{4}, k \in T_{3}\right)$. The decision matrixes can be denoted by $D^{k}=\left(\widetilde{\alpha}_{i j}^{k}\right)_{4 \times 4}\left(k \in T_{3}\right)$, and the weighting vector of $C_{j}\left(j \in N_{4}\right)$ is $\omega=$ $(0.2,0.15,0.35,0.3)^{T}$, and the weighting vector of $d_{k}\left(k \in T_{3}\right)$ is $\lambda=(0.5,0.3,0.2)^{T}$.

$$
\begin{aligned}
& D^{1}=\left(\begin{array}{l}
([0.3,0.5],[0.3,0.6])([0.2,0.7],[0.3,0.4]) \\
([0.3,0.6],[0.4,0.7])([0.4,0.7],[0.2,0.5]) \\
([0.4,0.6],[0.3,0.6])([0.5,0.7],[0.2,0.6]) \\
([0.5,0.6],[0.3,0.7])([0.5,0.7],[0.3,0.5])
\end{array}\right. \\
& ([0.2,0.4],[0.4,0.7])([0.1,0.3],[0.5,0.8])) \\
& ([0.2,0.5],[0.3,0.8])([0.4,0.5],[0.4,0.7]) \\
& ([0.3,0.6],[0.3,0.5])([0.2,0.3],[0.4,0.6]) \\
& ([0.3,0.5],[0.2,0.4])([0.5,0.5],[0.4,0.4])) \\
& D^{2}=\left(\begin{array}{l}
([0.1,0.4],[0.4,0.7])([0.2,0.5],[0.3,0.8]) \\
([0.4,0.6],[0.3,0.6]) \\
([0.3,0.5],[0.3,0.6])([0.3,0.8],[0.3,0.5]) \\
([0.3,0.6],[0.4,0.5])
\end{array}\right. \\
& ([0.3,0.7],[0.1,0.7])([0.1,0.2],[0.3,0.4])) \\
& ([0.2,0.4],[0.2,0.4])([0.2,0.6],[0.3,0.5]) \\
& ([0.3,0.5],[0.2,0.6])([0.2,0.3],[0.3,0.4]) \\
& ([0.4,0.7],[0.1,0.4])([0.5,0.6],[0.6,0.7])) \\
& D^{3}=\left(\begin{array}{l}
([0.3,0.4],[0.4,0.6])([0.2,0.5],[0.3,0.6]) \\
([0.3,0.3],[0.4,0.6])([0.2,0.6],[0.6,0.7]) \\
([0.3,0.4],[0.3,0.5])([0.2,0.5],[0.2,0.4]) \\
([0.3,0.6],[0.1,0.7])([0.2,0.6],[0.2,0.4])
\end{array}\right. \\
& ([0.2,0.4],[0.2,0.5])([0.2,0.3],[0.7,0.8])) \\
& ([0.1,0.4],[0.3,0.5])([0.2,0.4],[0.3,0.6]) \\
& ([0.2,0.3],[0.3,0.4])([0.1,0.3],[0.4,0.6]) \\
& ([0.5,0.8],[0.1,0.3])([0.4,0.7],[0.3,0.5]))
\end{aligned}
$$

The developed decision-making approach in this study is applied to derive the ordering relation of
$A_{i}\left(i \in M_{4}\right)$. The decision matrixes are listed as following and the implementation steps with details are provided subsequently.

Step 1. Utilize (47) $\sim(50)$ to obtain the weighting matrixes $\Phi^{k}=\left(\zeta_{i j}^{k}\right)_{4 \times 4}\left(k \in T_{3}\right)$ for the decision matrixes $D^{k}=\left(\widetilde{P}_{i j}^{k}\right)_{4 \times 4}\left(k \in T_{3}\right)$ :

$$
\begin{aligned}
\Phi^{1} & =\left(\begin{array}{llll}
0.5013 & 0.4949 & 0.4873 & 0.5027 \\
0.5009 & 0.4097 & 0.4796 & 0.4847 \\
0.4954 & 0.4800 & 0.4995 & 0.5064 \\
0.5040 & 0.4984 & 0.4921 & 0.5026
\end{array}\right), \\
\Phi^{2} & =\left(\begin{array}{llll}
0.2981 & 0.2975 & 0.3049 & 0.2980 \\
0.3039 & 0.2972 & 0.3096 & 0.3076 \\
0.3071 & 0.3134 & 0.3058 & 0.2910 \\
0.2940 & 0.3053 & 0.3102 & 0.2947
\end{array}\right), \\
\Phi^{3} & =\left(\begin{array}{llll}
0.2005 & 0.2075 & 0.2078 & 0.1993 \\
0.1952 & 0.1932 & 0.2108 & 0.2077 \\
0.1974 & 0.2066 & 0.1947 & 0.2026 \\
0.2020 & 0.1963 & 0.1976 & 0.2027
\end{array}\right) .
\end{aligned}
$$

Step 2. Use the IVPFFPWA operator (51) (let $\chi=2$ ) to fuse all the matrices $D^{k}=$ $\left(\widetilde{P}_{i j}^{k}\right)_{4 \times 4}\left(k \in T_{3}\right)$ to the collective matrix $D$.

$D=\left(\widetilde{P}_{i j}\right)_{4 \times 4}=$

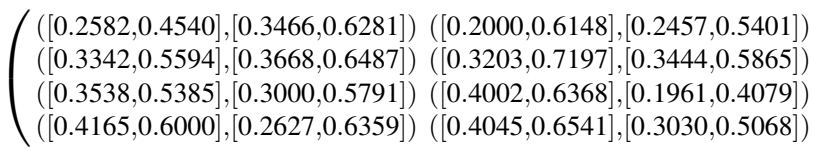

$([0.2355,0.4339],[0.2285,0.5537])([0.4000,0.5331],[0.3000,0.384]))$ $([0.1837,0.4518],[0.2648,0.5912])([0.3156,0.4523],[0.3452,0.4090])$ $([0.2836,0.5282],[0.2652,0.4790])([0.1843,0.3000],[0.3681,0.5348])$ $([0.3808,0.6482],[0.1408,0.3056])([0.4821,0.5802],[0.4270,0.4962]))$

Step 3. Aggregate all the values $\widetilde{P}_{i j}\left(j \in N_{4}\right)$ in matrix $D$ based on the IVPFFWA operator (53). Then obtain the overall values $\widetilde{P}_{i}\left(i \in M_{4}\right)$ corresponding to the alternative $A_{i}\left(i \in M_{4}\right)$ :

$$
\begin{aligned}
& \widetilde{P}_{1}=([0.2961,0.5015],[0.2726,0.5087]), \\
& \widetilde{P}_{2}=([0.2826,0.5301],[0.3185,0.5403]), \\
& \widetilde{P}_{3}=([0.2969,0.4994],[0.2870,0.5025]), \\
& \widetilde{P}_{4}=([0.4246,0.6206],[0.2507,0.4438]) .
\end{aligned}
$$

Step 4. By Definition 2, we calculate the score values $\operatorname{Sco}\left(\widetilde{P}_{i}\right)(i \in M)$ for $\widetilde{P}_{i}\left(i \in M_{4}\right)$ :

$$
\begin{aligned}
& \operatorname{Sco}\left(\widetilde{P}_{1}\right)=0.0031, \operatorname{Sco}\left(\widetilde{P}_{2}\right)=-0.0163, \\
& \operatorname{Sco}\left(\widetilde{P}_{3}\right)=0.0013, \operatorname{Sco}\left(\widetilde{P}_{4}\right)=0.1528 .
\end{aligned}
$$


Since $\operatorname{Sco}\left(\widetilde{P}_{4}\right)>\operatorname{Sco}\left(\widetilde{P}_{1}\right)>\operatorname{Sco}\left(\widetilde{P}_{3}\right)>$ $\operatorname{Sco}\left(\widetilde{P}_{2}\right)$, then $\widetilde{P}_{4} \succ \widetilde{P}_{1} \succ \widetilde{P}_{3} \succ \widetilde{P}_{2}$. Therefore, we have $A_{4} \succ A_{1} \succ A_{3} \succ A_{2}$. Thus, the best alternative is $A_{4}$.

From Step 1, it is convenient to find that different attribute values $\widetilde{P}_{i j}^{k}\left(i \in M_{4}, j \in N_{4}, k \in T_{3}\right)$ have different associated weights $\zeta_{i j}^{k}\left(i \in M_{4}, j \in N_{4}, k \in T_{3}\right)$, which is consists of the expert weight $\lambda_{k}$ and the support $\Delta\left(\widetilde{P}_{i j}^{k}\right)\left(i \in M_{4}, j \in N_{4}, k \in T_{3}\right)$. Then, the associated weight $\zeta_{i j}^{k}$ increases as the values of $\lambda_{k}$ or $\Delta\left(\widetilde{P}_{i j}^{k}\right)$ increase.

If we use the IVPFFPWG operator (52) (let $\chi=$ 2 ) instead of the IVPFFPWA to solve the above decision problem, then we obtain the collective values $\widetilde{P}_{i}^{\prime}\left(i \in M_{4}\right)$ corresponding to $A_{i}\left(i \in M_{4}\right)$ as follows:

$$
\begin{aligned}
\widetilde{P}_{1}^{\prime} & =([0.2620,0.4838],[0.3066,0.5594]), \\
\widetilde{P}_{2}^{\prime} & =([0.2454,0.4954],[0.3374,0.5981]), \\
\widetilde{P}_{3}^{\prime} & =([0.2620,0.4492],[0.3080,0.5199]), \\
\widetilde{P}_{4}^{\prime} & =([0.3998,0.6008],[0.3294,0.5076]) .
\end{aligned}
$$

Therefore, by Definition 2, we obtain the score values of $\widetilde{P}_{i}^{\prime}\left(i \in M_{4}\right)$, respectively:

$$
\begin{aligned}
& \operatorname{Sco}\left(\widetilde{P}_{1}^{\prime}\right)=-0.0523, \operatorname{Sco}\left(\widetilde{P}_{2}^{\prime}\right)=-0.0830, \\
& \operatorname{Sco}\left(\widetilde{P}_{3}^{\prime}\right)=-0.0484, \operatorname{Sco}\left(\widetilde{P}_{4}^{\prime}\right)=0.0785 . \\
& \operatorname{Sco}\left(\widetilde{P}_{4}^{\prime}\right)>\operatorname{Sco}\left(\widetilde{P}_{3}^{\prime}\right)>\operatorname{Sco}\left(\widetilde{P}_{1}^{\prime}\right)>\operatorname{Sco}\left(\widetilde{P}_{2}^{\prime}\right), \\
& \text { so } \widetilde{P}_{4}^{\prime} \succ \widetilde{P}_{3} \succ \widetilde{P}_{1}^{\prime} \succ \widetilde{P}_{2}^{\prime} . \text { Therefore } A_{4} \succ A_{3} \succ A_{1} \succ
\end{aligned}
$$

$A_{2}$. Thus, the best alternative is $A_{4}$.

From these results, with the same value of parameter $\chi=2$, we find that the IVPFFWA and IVPFFWG operators lead to different ranking positions of $A_{1}$ and $A_{3}$. However, the best alternative is always $A_{4}$. By comparing the score values $\operatorname{Sco}\left(\widetilde{P}_{i}\right)\left(i \in M_{4}\right)$ with $\operatorname{Sco}\left(\widetilde{P}_{i}^{\prime}\right)\left(i \in M_{4}\right)$, a useful observation is that the values $\widetilde{P}_{i}\left(i \in M_{4}\right)$ aggregated from the IVPFFWA operator are greater than those aggregated from IVPFFWG operator.

\subsection{Influence of parameter on aggregation operators}

An investigation on how the decision result changes with different choices of parameter $\chi$ in the above decision problem is offered in this section, and then, four descriptive figures will be provided to intensify the understanding of our proposal. For the sake of convenience, if the IVPFFPWA operator is used as the aggregation tool for the decision process, then we denote $\widetilde{P}_{i}^{A}\left(i \in M_{4}\right)$ as the collective values of $A_{i}\left(i \in M_{4}\right)$. Similarly, if the used tool is the IVPFFPWG operator, then $\widetilde{P}_{i}^{G}\left(i \in M_{4}\right)$ is denoted as the collective values.

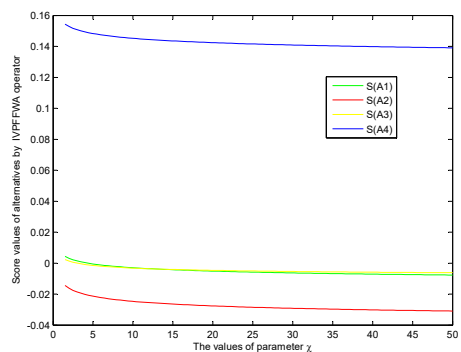

Fig. 1. Score values obtained in the use of the IVPFFPWA operator.

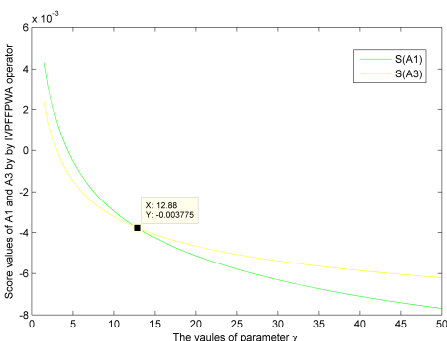

Fig. 2. Score values of $A_{1}$ and $A_{3}$ obtained in the use of the IVPFFPWA operator.

Figure 1 shows how the score values given by IVPFFPWA operator decrease according to the increasing $\chi$. Moreover, from Figure 1, the following cases can be obtained:

(1) If $\chi \in(1,12.88]$, then the order relation of alternatives $A_{i}\left(i \in M_{4}\right)$ is $A_{4} \succ A_{1} \succ A_{3} \succ A_{2}$, and the best alternative is $A_{4}$. If $\chi \in(12.88,50)$, then we obtain the ordering relation of $A_{i}\left(i \in M_{4}\right)$ as $A_{4} \succ A_{3} \succ A_{1} \succ A_{2}$.

(2) The score values $\operatorname{Sco}\left(\widetilde{P}_{i}^{A}\right)\left(i \in M_{4}\right)$ for the alternatives $A_{i}\left(i \in M_{4}\right)$ decrease with respect to $\chi$.

The score value $\operatorname{Sco}\left(\widetilde{P}_{1}^{A}\right)$ and that of $\operatorname{Sco}\left(\widetilde{P}_{3}^{A}\right)$ are pretty close, therefore, we provide more details for them, which are shown in Figure 2.

(i) If $\chi \in(1,12.88)$, then $\operatorname{Sco}\left(\widetilde{P}_{1}^{A}\right)>\operatorname{Sco}\left(\widetilde{P}_{3}^{A}\right)$. Thus, $\widetilde{P}_{1}^{A} \succ \widetilde{P}_{3}^{A}$. 
(ii) If $\chi=12.88$, then $\operatorname{Sco}\left(\widetilde{P}_{1}^{A}\right)=\operatorname{Sco}\left(\widetilde{P}_{3}^{A}\right)=$ -0.0038 and $\operatorname{Acc}\left(\widetilde{P}_{1}^{A}\right)=0.3396>\operatorname{Acc}\left(\widetilde{P}_{3}^{A}\right)=$ 0.3352. Thus, $\widetilde{P}_{1}^{A} \succ \widetilde{P}_{3}^{A}$.

(iii) If $\chi \in(12.88,50)$, then $\operatorname{Sco}\left(\widetilde{P}_{1}^{A}\right)<\operatorname{Sco}\left(\widetilde{P}_{3}^{A}\right)$. Thus, $\widetilde{P}_{1}^{A} \prec \widetilde{P}_{3}^{A}$.

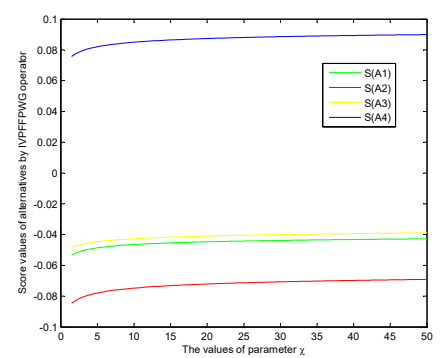

Fig. 3. Score values obtained in the use of the IVPFFPWG operator.

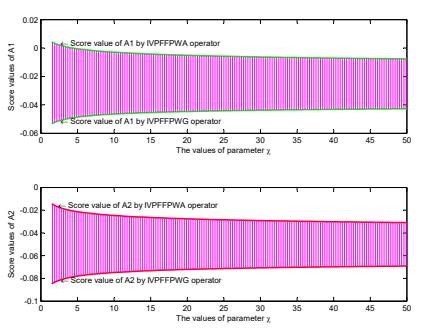

Fig. 4. Score values of $A_{1}$ and $A_{2}$ obtained in the use of the IVPFFPWA/IVPFFPWG operator.

Figure 3 illustrates how the score values obtained with the IVPFFPWG operator increase in respect of the increasing $\chi$, and more analysis are offered as following.

(1) When $\chi \in(0,50)$, the ranking of $A_{i}\left(i \in M_{4}\right)$ is $A_{4} \succ A_{3} \succ A_{1} \succ A_{2}$, and the best alternative is invariably $A_{4}$.

(2) The score values $\operatorname{Sco}\left(\widetilde{P}_{i}^{G}\right)\left(i \in M_{4}\right)$ for the alternatives $A_{i}\left(i \in M_{4}\right)$ increase with respect to $\chi$.
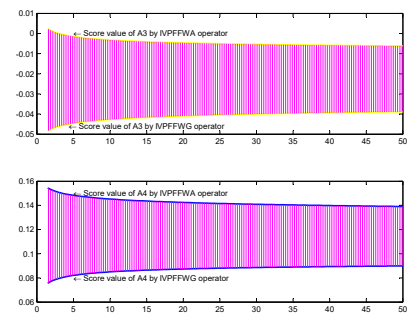

Fig. 5. Score values of $A_{3}$ and $A_{4}$ obtained in the use of the IVPFFPWA/IVPFFPWG operator.

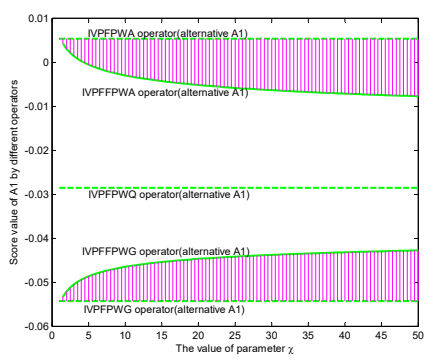

Fig. 6. Score values of $A_{1}$ by different operators.

Figures 4 and 5 show the deviations between the score values obtained by the IVPFFPWA operator and those obtained by the IVPFFPWG operator, from which we obtain $\operatorname{Sco}\left(\widetilde{P}_{i}^{G}\right)<$ $\operatorname{Sco}\left(\widetilde{P}_{i}^{A}\right)\left(i \in M_{4}\right)$. In addition, the values of $\operatorname{Sco}\left(\widetilde{P}_{i}^{A}\right)-\operatorname{Sco}\left(\widetilde{P}_{i}^{G}\right)\left(i \in M_{4}\right)$ become smaller as the value of $\chi$ increases. It illustrates that the aggregation result calculated by the IVPFFPWG operator is smaller than the result obtained from the IVPFFPWA operator. Therefore, the IVPFFPWA operator is suitable for modeling optimistic DMs, whereas the IVPFFPWG operator is considered to be useful in reflecting pessimistic DMs. According to Figures 1 and 3 , we obtain that the smaller $\chi$ gets, the greater the level of optimism and pessimism will be.

We use two generalized operators (IVPFFPWA/IVPFFPWG) and their limiting operators (IVPFPWA/IVPFPWQ/IVPFPWG) to obtain the score values of alternative $A_{1}$. The details can be found in Figure 6. Consequently, the following ordering relation is obtained from Figure 6: $\operatorname{Sco}\left(\widetilde{P}_{1}^{I V P F P W G}\right)<\operatorname{Sco}\left(\widetilde{P}_{1}^{G}\right)<\operatorname{Sco}\left(\widetilde{P}_{1}^{I V P F P W Q}\right)<$ $\operatorname{Sco}\left(\widetilde{P}_{1}^{A}\right)<\operatorname{Sco}\left(\widetilde{P}_{1}^{I V P F P W A}\right)$.

Figure 6 illustrates the level of optimism and pessimism decreases when $\chi$ increases, and the decision maker's attitude could be regarded as neutral when $\chi \rightarrow \infty$.

According to the previous analysis, it is observed that the associated parameter $\chi$ to the IVPFFPWG and the IVPFFPWA operators can be considered as a promising reflection of the attitude of DM. The DMs can obtain different score values of the collective overall preference values when different values of parameter $\chi$ are fixed in the sense that they can 
obtain different rankings of alternatives indicating their preferences. Therefore, the decision-making approaches with the IVPFFPWG and the IVPFFPWA operators are highly flexible and can provide DMs with more choices in handling different reallief scenarios.

\subsection{Comparative analysis}

In the sequel, the proposed IVPFFP aggregation operators will be compared to several aggregation operators that were developed in $10,13,16$ to evidence their superiority. Detailed analysis are provided in accordance with the performance of the compared aggregation operators given that they are used for the aggregation of individual decision matrices $D^{k}(k \in T)$ in the context of MAGDM.

The three selected aggregation operators for comparison are briefly introduced in the first place. In Peng and Yang ${ }^{10}$, the weighted average (WA) and weighted geometric (WG) operators were accommodated to the Pythagorean fuzzy environment. The interval-valued Pythagorean fuzzy WA operator and WG operator, which are denoted separately by P-IVPFWA and the P-IVPFWG to distinguish themselves from the proposed ones, were developed to aggregate individual IVPF decision matrices. In Liang et al. ${ }^{13}$, based on the Algebraic operational laws ${ }^{10,11}$, the IVPFWA operator and IVPFWG operator were defined to aggregate individual decision matrices into a collective decision matrix. In Zhang ${ }^{16}$, the frank t-norm and s-norm were adopted as a basis for defining the interval-valued intuitionistic fuzzy frank weighted average (IVIFFWA) operator and the interval-valued intuitionistic fuzzy frank weighted geometric (IVIFFWG) operator, which were further used in the aggregation of individual IVIF decision matrixes.

The first comparison made is between the PIVPFWA and P-IVPFWG operators and the IVPFFP operator. Recall that the generalized Pythagorean fuzzy aggregation operator was defined by Yager $^{1,2}$ to gather a collection of PFNs satisfying the following characteristic: $A g g=\eta_{p} \circ A g g^{d} \circ \eta_{p}$, where Agg and $A g g^{d}$ are dual with respect to the Pythagorean negation $\eta_{p}(x)=\sqrt{1-x^{2}}$, and $A g g$ and $A g g^{d}$ are the membership and non-membership functions, re- spectively. According to Theorem 21, it is evident that the IVPFFP operator proposed in this paper satisfies this characteristic as well. However, the PIVPFWA and P-IVPFWG operators fail to meet the dual property as their related aggregation functions were weighted average (WA) operator $\left(\sum_{i=1}^{n} w_{i} x_{i}\right)$ and weighted geometric (WG) operator $\left(\prod_{i=1}^{n} x_{i}^{w_{i}}\right)$. Subsection 6.1 is used as the background for case study, and applying the P-IVPFWA and P-IVPFWG operators derive the following decision results.

(i) In the case that the P-IVPFWA operator was applied we have $\operatorname{Sco}\left(\widetilde{P}_{1}\right)=-0.0226, \operatorname{Sco}\left(\widetilde{P}_{2}\right)=$ -0.0479 and $\operatorname{Sco}\left(\widetilde{P}_{3}\right)=-0.0252, \operatorname{Sco}\left(\widetilde{P}_{4}\right)=$ 0.1156 . Therefore, $\operatorname{Sco}\left(\widetilde{P}_{4}\right)>\operatorname{Sco}\left(\widetilde{P}_{1}\right)>\operatorname{Sco}\left(\widetilde{P}_{3}\right)>$ $\operatorname{Sco}\left(\widetilde{P}_{2}\right)$. Thus, the ranking of alternatives is $A_{4} \succ$ $A_{1} \succ A_{3} \succ A_{2}$, which is consistent with the result obtained by IVPFFPWA operator $(\chi \in(1,12.88])$ in subsection 6.2.

(ii) In the other case that the P-IVPFWG operator was applied we obtain $\operatorname{Sco}\left(\widetilde{P}_{1}\right)=$ $-0.0221, \operatorname{Sco}\left(\widetilde{P}_{2}\right)=-0.0479$ and $\operatorname{Sco}\left(\widetilde{P}_{3}\right)=$ $-0.0329, \operatorname{Sco}\left(\widetilde{P}_{4}\right)=0.1287$. Therefore, $\operatorname{Sco}\left(\widetilde{P}_{4}\right)>$ $\operatorname{Sco}\left(\widetilde{P}_{1}\right)>\operatorname{Sco}\left(\widetilde{P}_{3}\right)>\operatorname{Sco}\left(\widetilde{P}_{2}\right)$. Thus, the ranking of alternatives is $A_{4} \succ A_{1} \succ A_{3} \succ A_{2}$, which is consistent with the result obtained by IVPFFPWA operator $(\chi \in(1,12.88])$ in subsection 6.2.

The second comparison will be conducted for the IVIFFWA and IVIFFWG operators. According to Theorem 23, the IVPFWA and IVPFWG operators are essentially the respective special cases of the IVPFPWA and IVPFPWG operators. Likewise, the IVPFWA and IVPFWG operators are adopted to address the MAGDM problem in subsection 6.1 and the following decision results can be obtained.

(i) In the case that the IVPFWA operator was applied we have $\operatorname{Sco}\left(\widetilde{P}_{1}\right)=0.0054, \operatorname{Sco}\left(\widetilde{P}_{2}\right)=$ $-0.0134, \operatorname{Sco}\left(\widetilde{P}_{3}\right)=0.0043, \operatorname{Sco}\left(\widetilde{P}_{4}\right)=0.1556$. Therefore, $\operatorname{Sco}\left(\widetilde{P}_{4}\right)>\operatorname{Sco}\left(\widetilde{P}_{1}\right)>\operatorname{Sco}\left(\widetilde{P}_{3}\right)>\operatorname{Sco}\left(\widetilde{P}_{2}\right)$. Thus, $A_{4} \succ A_{1} \succ A_{3} \succ A_{2}$, which is consistent with the result obtained by IVPFFPWA operator $(\chi \in$ $(1,12.88])$ in subsection 6.2.

(ii) In the other case that the IVPFWG operator was considered we haveSco $\left(\widetilde{P}_{1}\right)=$ $-0.0562, \operatorname{Sco}\left(\widetilde{P}_{2}\right)=-0.0891$ and $\operatorname{Sco}\left(\widetilde{P}_{3}\right)=$ $-0.0495, \operatorname{Sco}\left(\widetilde{P}_{4}\right)=0.0721$. Therefore, $\operatorname{Sco}\left(\widetilde{P}_{4}\right)>$ 
$\operatorname{Sco}\left(\widetilde{P}_{3}\right)>\operatorname{Sco}\left(\widetilde{P}_{1}\right)>\operatorname{Sco}\left(\widetilde{P}_{2}\right)$. Thus, $A_{4} \succ A_{3} \succ$ $A_{1} \succ A_{2}$, which is consistent with the result obtained by IVPFFPWG operator $(\chi \in(1,50))$ in subsection 6.2 .

The last comparison was made between Zhang 16 and our proposal. Yager ${ }^{1,2}$ points out that all IFNs are PFNs, but not vice versa. Likewise, all IVIFNs are IVPFNs, but not the other way around. Therefore, the IVIFFWA and IVIFFWG operators in ${ }^{16}$ can not be used to solve the aforementioned MAGDM problem. On the contrary, the IVPFFP operator proposed in this paper is capable of addressing the MAGDM problem provided as Example 5.1 in ${ }^{16}$, and the following decision results can be obtained accordingly.

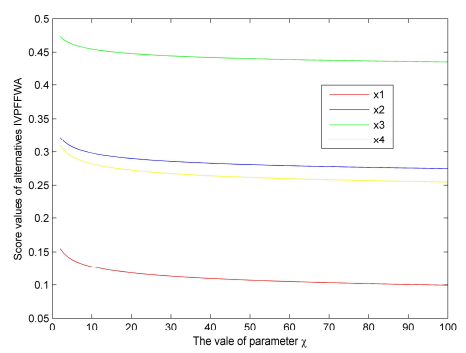

Fig. 7. Score values by IVPFFPWA operators.

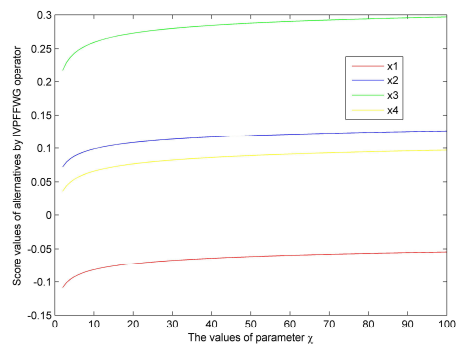

Fig. 8. Score values by IVPFFPWG operators.

(i) If the IVPFFPWA operator instead of the IVIFFWA operator has been adopted, then we have $\operatorname{Sco}\left(\widetilde{r}_{3}\right)>\operatorname{Sco}\left(\widetilde{r}_{2}\right)>\operatorname{Sco}\left(\widetilde{r}_{4}\right)>\operatorname{Sco}\left(\widetilde{r}_{1}\right), \chi \in$ $(1,100]$. Thus, the ranking of alternatives is $x_{3} \succ$ $x_{2} \succ x_{4} \succ x_{1}$. More details can be found in Figure 7. Although the ranking of alternative $x_{2}$ and alternative $x_{4}$ is different from that obtained from the IVIFFWA operator, the best alternative remains $x_{3}$.

(ii) If the IVPFFPWG operator rather than the IVIFFWG operator has been adopted, then we get $\operatorname{Sco}\left(\widetilde{r}_{3}\right)>\operatorname{Sco}\left(\widetilde{r}_{2}\right)>\operatorname{Sco}\left(\widetilde{r}_{4}\right)>\operatorname{Sco}\left(\widetilde{r}_{1}\right), \chi \in$ $(1,100]$. Thus, the ranking of alternatives is $x_{3} \succ$ $x_{2} \succ x_{4} \succ x_{1}$.
The final score values changing with the varying parameter are shown in Figure 8. Despite the ranking of alternatives $x_{2}$ and $x_{4}$ obtained using our approach is different from that derived from the IVIFFWG operator, the best alternative selected is $x_{3}$ in both cases. The reason that the ranking positions of $\left(x_{2}\right.$ and $\left.x_{4}\right)$ get changed is because the IVPFFPWA and IVPFFPWG operators take into account the support function, and the scores of alternatives $x_{2}$ and $x_{4}$ are pretty close to each other. This is generally not the case for the IVIFFWA and IVIFFWG operators as the support function was not factored in.

In comparison to the several existing aggregation operators described above, the proposed IVPFFP aggregation operators present the following benefits in its implementation process.

(1) The convenience of expert weight elicitation.

The IVPFFP operator can provide DM more flexibility in determining the weights of experts in the context of MAGDM. On the one hand, if the DM trusts the expert who provides evaluations completely and is allowed to determine the weight of the expert all on his/her own, then the support degree can be set as a constant, in which case the IVPFFPWA and IVPFFPWG operators degenerate to the IVPFFWA and IVPFFWG operator, respectively. This is a fact that can be reflected from Theorem 13(2) and Theorem 17(2). On the other hand, if the DM gets inadequate information at hand about the expert who provides evaluations, the expert weight elicitation depends entirely on the support function. In this case, the IVPFFPWA and IVPFFPWG operators reduce to the IVPFFPA and IVPFFPG operators, respectively, which can be observed in Theorem 13(1) and Theorem 17(1). Otherwise, the combination weight involving both subjective and objective approaches can be used to determine the aggregation weight of experts with the IVPFFWA and IVPFFWG operators.

(2) The variable parameter values indicating preference orientations.

Following the previous analysis conducted in subsection 6.2, it is observed that the adjustable parameter $\chi$ conforms to the DM's preferences in the sense that the DM can determine the appropriate values of $\chi$ in accordance with their preference orien- 
tations.

(3)The expansion of domain for evaluation.

The IVPFFPWA and IVPFFPWG operators are able to deal with MAGDM problems with intervalvalued Pythagorean fuzzy inputs, which is a benefit that the IVPFFWA and IVPFFWG operator do not share. It is as well convenient for DMs to adapt the IVPFFPWA and IVPFFPWG operators into MAGDM with interval-valued intuitionistic fuzzy inputs in the use of the idea raised in this paper.

\section{Conclusions}

In this study, we applied a special automorphism on the unit interval to construct an isomorphic Frank dual triple, which can be used to define the intervalvalued Pythagorean Frank operations. We further revealed that these generalized operations include the existing operations for IVPFNs as special cases and discussed some fundamental properties of them. Subsequently, we proposed the IVPFFPWA and IVPFFPWG operators based on the proposed Frank operations and explored plenty of instrumental properties of the IVPFFPWA and IVPFFPWG operators. Several limiting cases of the proposed aggregation operators have as well been discussed in respect of the introduced adjustable parameter. We developed an IVPFFPWA (or IVPFFPWG) operatorbased technique to deal with a classical MAGDM problem, provided an illustrative example to effectively verify the approach, and studied the influences of the adjustable parameter on the final aggregation results. The comparative analysis further demonstrated the superiority of the proposed aggregation techniques.

In future study, we are poised to pay more attention to the integration of IVPFNs with linguistic implication to foster their applications in the area of linguistic decision making ${ }^{33,34}$. Investigation on how varying associated weighting vectors will impact the final decision outputs under the intervalvalued Pythagorean fuzzy environment will as well be a promising endeavor for future research ${ }^{35}$. Certain accuracy enhancements of the MAGDM with IVPFNs are expected to be achieved in the subse- quent development of this study.

\section{Acknowledgments}

This work was supported by "the Fundamental Research Funds for the Central Universities" (Grant No. 2042018kf0006) and the Theme-based Research Projects of the Research Grants Council (RGC) (Grant No. T32-101/15-R), and partly supported by the Key Project of the National Natural Science Foundation of China (Grant No. 71231007), the National Natural Science Foundation of China (Grant No. 71373222), and the Open Fund of Key Laboratory of Hunan Province (2017TP1026).

\section{References}

1. Yager R R, Abbasov A M. Pythagorean membership grades, complex numbers, and decision making. International Journal of Intelligent Systems, 2013, 28(5): 436-452.

2. Yager R R. Pythagorean membership grades in multicriteria decision making. IEEE Transactions on Fuzzy Systems, 2014, 22(4): 958-965.

3. Atanassov K T. Intuitionistic fuzzy sets. Fuzzy sets and Systems, 1986, 20(1): 87-96.

4. Beliakov G, James S. Averaging aggregation functions for preferences expressed as Pythagorean membership grades and fuzzy orthopairs. Fuzzy Systems (FUZZ-IEEE), 2014 IEEE International Conference on. IEEE, 2014: 298-305.

5. Zhang X, Xu Z. Extension of TOPSIS to multiple criteria decision making with Pythagorean fuzzy sets. International Journal of Intelligent Systems, 2014, 29(12): 1061-1078.

6. Peng X, Yang Y. Some results for Pythagorean fuzzy sets. International Journal of Intelligent Systems, 2015, 30(11): 1133-1160.

7. Garg H. A new generalized pythagorean fuzzy information aggregation using einstein operations and its application to decision making. International Journal of Intelligent Systems, 2016, 31(9): 886-920.

8. Peng X, Dai J. Approaches to Pythagorean Fuzzy Stochastic Multi-criteria Decision Making Based on Prospect Theory and Regret Theory with New Distance Measure and Score Function. International Journal of Intelligent Systems, 2017.

9. Peng X, Yuan H, Yang Y. Pythagorean fuzzy information measures and their applications. International Journal of Intelligent Systems, 2017.

10. Peng X, Yang Y. Fundamental Properties of IntervalValued Pythagorean Fuzzy Aggregation Operators. 
International Journal of Intelligent Systems, 2016, 31(5): 444-487.

11. Zhang X. Multicriteria Pythagorean fuzzy decision analysis: a hierarchical QUALIFLEX approach with the closeness index-based ranking methods. Information Sciences, 2016, 330: 104-124.

12. Garg H. A novel accuracy function under intervalvalued pythagorean fuzzy environment for solving multi-criteria decision making problem. Journal of Intelligent \& Fuzzy Systems, 2016, 31(1): 529-540.

13. Liang $\mathrm{W}$, Zhang $\mathrm{X}$, Liu $\mathrm{M}$. The maximizing deviation method based on interval-valued Pythagorean fuzzy weighted aggregating operator for multiple criteria group decision analysis. Discrete Dynamics in Nature and Society, 2015, 2015.

14. Xia M, Xu Z, Zhu B. Some issues on intuitionistic fuzzy aggregation operators based on Archimedean t-conorm and t-norm. Knowledge-Based Systems, 2012, 31: 78-88.

15. Zhang X, Liu P, Wang Y. Multiple attribute group decision making methods based on intuitionistic fuzzy frank power aggregation operators. Journal of Intelligent \& Fuzzy Systems, 2015, 29(5): 2235-2246.

16. Zhang Z. Interval-valued intuitionistic fuzzy Frank aggregation operators and their applications to multiple attribute group decision making. Neural Computing and Applications, 2017, 28(6): 1471-1501.

17. Qin J, Liu X, Pedrycz W. Frank aggregation operators and their application to hesitant fuzzy multiple attribute decision making. Applied Soft Computing, 2016, 41: 428-452.

18. Qin J, Liu X. Frank aggregation operators for triangular interval type-2 fuzzy set and its application in multiple attribute group decision making. Journal of Applied Mathematics, 2014, 2014.

19. Du Y, Hou F. Interval intuitionistic linguistic Frank aggregation operators and their application in decision making. Control and Decision, 2017.

20. Wang L, Shen Q, Zhu L. Dual hesitant fuzzy power aggregation operators based on Archimedean $\mathrm{t}$-conorm and t-norm and their application to multiple attribute group decision making. Applied Soft Computing, 2016, 38: 23-50.

21. Frank M J. On the simultaneous associativity of $F(x, y)$ and $x+y-F(x, y)$. Aequationes mathematicae, 1979, 19(1):194-226.

22. $\mathrm{Xu} \mathrm{Z}$. Approaches to multiple attribute group decision making based on intuitionistic fuzzy power aggregation operators. Knowledge-Based Systems, 2011, 24(6): 749-760.

23. Zhang Z. Generalized Atanassov's intuitionistic fuzzy power geometric operators and their application to multiple attribute group decision making. Information Fusion, 2013, 14(4): 460-486.

24. Zhu C, Zhu L, Zhang X. Linguistic hesitant fuzzy power aggregation operators and their applications in multiple attribute decision-making. Information Sciences, 2016, 367: 809-826.

25. Liu P, Liu X. The neutrosophic number generalized weighted power averaging operator and its application in multiple attribute group decision making. International Journal of Machine Learning and Cybernetics, 2016: 1-12.

26. Yager R R. The power average operator. IEEE Transactions on Systems, Man, and Cybernetics-Part A: Systems and Humans, 2001, 31(6): 724-731.

27. Xu Z, Yager R R. Power-geometric operators and their use in group decision making. IEEE Transactions on Fuzzy Systems, 2010, 18(1): 94-105.

28. Klement E P, Mesiar R, Pap E. Triangular Norms. Springer Netherlands, 2000.

29. Beliakov G, Pradera A, Calvo T. Aggregation functions: A guide for practitioners. Heidelberg: Springer, 2007.

30. Herrera F, Herrera-Viedma E. Linguistic decision analysis: steps for solving decision problems under linguistic information. Fuzzy Sets and systems, 2000, 115(1): 67-82.

31. Chen Z S, Chin K S, Li Y L, Yang Y. On generalized extended Bonferroni means for decision making. IEEE Transactions on Fuzzy Systems, 2016, 24(6): 1525-1543.

32. Xiong $\mathrm{S} \mathrm{H}$, Chen Z S, Li Y L, Chin K S. On extending power-geometric operators to interval-valued hes- itant fuzzy sets and their applications to group decision making. International Journal of Information Technology and Decision Making, 2016, 15(05): 1055-1114.

33. Rodríguez R M, Labella A, Martínez L. An overview on fuzzy modelling of complex linguistic preferences in decision making. International Journal of Computational Intelligence Systems, 2016 9(sup1), 81-94.

34. Martínez L, Rodríguez R M, Herrera F, The 2-tuple Linguistic Model: Computing with Words in Decision Making, Springer, New York, 2015.

35. Dong Y C, Liu Y T, Liang H M, Chiclana F, HerreraViedma, E. Strategic weight manipulation in multiple attribute decision making. Omega, 2018, 75: 154-164.

36. Yang Y, Ding H, Chen Z S, Li Y L, A note on extension of TOPSIS to multiple criteria decision making with Pythagorean fuzzy sets. International Journal of Intelligent Systems, 2016, 31(1): 68-72. 